\title{
Electronic structure of kinetic energy driven superconductors in the presence of bilayer splitting
}

\author{
Yu Lan, ${ }^{1}$ Jihong Qin, ${ }^{2}$ and Shiping Feng ${ }^{1}$ \\ ${ }^{1}$ Department of Physics, Beijing Normal University, Beijing 100875, China \\ ${ }^{2}$ Department of Physics, Beijing University of Science and Technology, Beijing 100083, China
}

(Dated: November 17, 2018)

\begin{abstract}
Within the framework of the kinetic energy driven superconductivity, the electronic structure of bilayer cuprate superconductors in the superconducting state is studied. It is shown that the electron spectrum of bilayer cuprate superconductors is split into the bonding and antibonding components by the bilayer splitting, then the observed peak-dip-hump structure around the $[\pi, 0]$ point is mainly caused by this bilayer splitting, with the superconducting peak being related to the antibonding component, and the hump being formed by the bonding component. The spectral weight increases with increasing the doping concentration. In analogy to the normal state case, both electron antibonding peak and bonding hump have the weak dispersions around the $[\pi, 0]$ point.
\end{abstract}

PACS numbers: 74.20.Mn, 74.20.-z, 74.25.Jb

\section{INTRODUCTION}

The parent compounds of cuprate superconductors are the Mott insulators with an antiferromagnetic (AF) longrange order (AFLRO), then via the charge carrier doping, one can drive these materials through a metal-insulating transition and enter the superconducting ( $\mathrm{SC}$ ) dome ${ }^{1,2,3}$. It has become clear in the past twenty years that cuprate superconductors are among the most complex systems studied in condensed matter physics ${ }^{1,2,3}$. The complications arise mainly from (1) a layered crystal structure with one or more $\mathrm{CuO}_{2}$ planes per unit cell separated by insulating layers which leads to a quasi-two-dimensional electronic structure, and (2) extreme sensitivity of the physical properties to the compositions (stoichiometry) which control the carrier density in the $\mathrm{CuO}_{2}$ plane ${ }^{1,2,3}$. As a consequence, both experimental investigation and theoretical understanding are extremely difficult.

By virtue of systematic studies using the angle-resolved photoemission spectroscopy (ARPES), the low-energy electronic structure of cuprate superconductors in the $\mathrm{SC}$ state is well-established by now ${ }^{2,3}$, where an agreement has emerged that the electronic quasiparticle-like excitations are well defined, and are the entities participating in the SC pairing. In particular, the lowest energy states are located at the $[\pi, 0]$ point of the Brillouin zone, where the d-wave SC gap function is maximal, then the most contributions of the electron spectral function come from the $[\pi, 0]$ point $^{2,3}$. Moreover, some ARPES experimental results unambiguously established the Bogoliubov-quasiparticle nature of the sharp SC quasiparticle peak near the $[\pi, 0]$ point ${ }^{4,5}$, then the $\mathrm{SC}$ coherence of the quasiparticle peak is described by the simple Bardeen-Cooper-Schrieffer (BCS) formalism ${ }^{6}$. However, there are numerous anomalies for different families of cuprate superconductors, which complicate the physical properties of the electronic structure ${ }^{2,3}$. Among these anomalies is the dramatic change in the spectral lineshape around the $[\pi, 0]$ point first observed on the bilayer cuprate superconductor $\mathrm{Bi}_{2} \mathrm{Sr}_{2} \mathrm{CaCu}_{2} \mathrm{O}_{8+\delta}$, where a sharp quasiparticle peak develops at the lowest binding energy, followed by a dip and a hump, giving rise to the so-called peak-dip-hump (PDH) structure in the elec- tron spectrum $7,8,9$. Later, this $\mathrm{PDH}$ structure was also found in $\mathrm{YBa}_{2} \mathrm{Cu}_{3} \mathrm{O}_{7-\delta} \frac{10}{}$ and in $\mathrm{Bi}_{2} \mathrm{Sr}_{2} \mathrm{Ca}_{2} \mathrm{Cu}_{3} \mathrm{O}_{10+\delta} 11$. Furthermore, although the sharp quasiparticle peaks are identified in the SC state along the entire Fermi surface, the PDH structure is most strongly developed around the $[\pi, 0]$ point $2,7,8,9,10,11$.

The appearance of the $\mathrm{PDH}$ structure in bilayer cuprate superconductors in the SC state is the mostly remarkable effect, however, its full understanding is still a challenging issue. The earlier works ${ }^{2,12}$ gave the main impetus for a phenomenological description of the singleparticle excitations in terms of an interaction between quasiparticles and collective modes, which is of fundamental relevance to the nature of superconductivity and the pairing mechanism in cuprate superconductors. However, the different interpretive scenario has been proposed $^{2,13}$. This followed from the observation of the bilayer splitting (BS) for both normal and SC states in a wide doping range ${ }^{14,15,16}$. This $\mathrm{BS}$ of the $\mathrm{CuO}_{2}$ plane derives the electronic structure in the bonding and antibonding bands due to the present of $\mathrm{CuO}_{2}$ bilayer blocks in the unit cell of bilayer cuprate superconductors, then the main features of the PDH structure is caused by the $\mathrm{BS}^{13,14,15,16}$, with the peak and hump corresponding to the antibonding and bonding bands, respectively. Furthermore, some ARPES experimental data measured above and below the SC transition temperature show that this PDH structure is totally unrelated to superconductivity ${ }^{14}$. The recent ARPES experimental results reported by several groups support this scenario, and most convincingly suggested that the $\mathrm{PDH}$ structure originates from the BS at any doping levels 17 . To the best of our knowledge, this PDH structure in bilayer cuprate superconductors has not been treated starting from a microscopic SC theory.

Within the single layer $t-t^{\prime}-J$ model, the electronic structure of the single layer cuprate superconductors in the $\mathrm{SC}$ state has been discussed ${ }^{18}$ based on the framework of the kinetic energy driven superconductivity ${ }^{19}$, and the main features of the ARPES experiments on the single layer cuprate superconductors have been reproduced, including the doping and temperature dependence of the electron spectrum and quasiparticle dispersion. In 
this paper, we study the electronic structure of bilayer cuprate superconductors in the SC state along with this line. Within the kinetic energy driven SC mechanism $\frac{19}{}$, we employed the $t-t^{\prime}-J$ model by considering the bilayer interaction, and then show explicitly that the BS occurs due to this bilayer interaction. In this case, the electron spectrum is split into the bonding and antibonding components by this BS, then the SC peak is closely related to the antibonding component, while the hump is mainly formed by the bonding component. In other words, the well pronounced PDH structure in the electron spectrum of bilayer cuprate superconductors is mainly caused by the BS. Furthermore, the spectral weight in the $[\pi, 0]$ point increases with increasing the doping concentration. In analogy to the normal-state case $\frac{14,20,21,22}{1,}$ both electron antibonding peak and bonding hump have the weak dispersions around the $[\pi, 0]$ point, in qualitative agreement with the experimental observation on bilayer cuprate superconductors in the SC state $2,7,8,9,10,11$.

The paper is organized as follows. The basic formalism is presented in Sec. II, where we generalize the kinetic energy driven superconductivity from the previous single layer case $\mathrm{1}^{18,19}$ to the bilayer case, and then evaluate explicitly the longitudinal and transverse components of the electron normal and anomalous Green's functions (hence the bonding and antibonding electron spectral functions). Within this theoretical framework, we discuss the electronic structure of bilayer cuprate superconductors in the SC state in Sec. III. It is shown that the striking PDH structure in bilayer cuprate superconductors is closely related to the BS. Finally, we give a summary and discussions in Sec. IV.

\section{FORMALISM}

It has been shown from the ARPES experiments,2,23 that the two-dimensional $t-t^{\prime}-J$ model is of particular relevance to the low energy features of cuprate superconductors. For discussions of the physical properties of bilayer cuprate superconductors, the $t-t^{\prime}-J$ model can be expressed by including the bilayer interactions as,

$$
\begin{aligned}
H & =-t \sum_{i \hat{\eta} a \sigma} C_{i a \sigma}^{\dagger} C_{i+\hat{\eta} a \sigma}+t^{\prime} \sum_{i \hat{\tau} a \sigma} C_{i a \sigma}^{\dagger} C_{i+\hat{\tau} a \sigma} \\
& -\sum_{i \sigma} t_{\perp}(i)\left(C_{i 1 \sigma}^{\dagger} C_{i 2 \sigma}+H . c .\right)+\mu \sum_{i a \sigma} C_{i a \sigma}^{\dagger} C_{i a \sigma} \\
& +J \sum_{i \hat{\eta} a} \mathbf{S}_{i a} \cdot \mathbf{S}_{i+\hat{\eta} a}+J_{\perp} \sum_{i} \mathbf{S}_{i 1} \cdot \mathbf{S}_{i 2}
\end{aligned}
$$

supplemented by an important on-site local constraint $\sum_{\sigma} C_{i a \sigma}^{\dagger} C_{i a \sigma} \leq 1$ to avoid the double occupancy, where $\hat{\eta}= \pm \hat{x}, \pm \hat{y}$ representing the nearest neighbors of a given site $i, \hat{\tau}= \pm \hat{x} \pm \hat{y}$ representing the next nearest neighbors of a given site $i, a=1,2$ is plane index, $C_{i a \sigma}^{\dagger}\left(C_{i a \sigma}\right)$ is the electron creation (annihilation) operator, $\mathbf{S}_{i a}=$ $C_{i a}^{\dagger} \sigma C_{i a} / 2$ is the spin operator with the Pauli matrices $\boldsymbol{\sigma}=\left(\sigma_{x}, \sigma_{y}, \sigma_{z}\right), \mu$ is the chemical potential, and the interlayer coherent hopping has the form,

$$
t_{\perp}(\mathbf{k})=\frac{t_{\perp}}{4}\left(\cos k_{x}-\cos k_{y}\right)^{2}
$$

which is strongly anisotropic and follows the theoretical predictions 24 . In particular, this momentum dependent form (2) has been experimentally verified 14.15 . For this $t-t^{\prime}-J$ model (1), it has been argued that crucial requirement is to impose the electron single occupancy local constraint for a proper understanding of the physical properties of cuprate superconductors. To incorporate the electron single occupancy local constraint, the charge-spin separation (CSS) fermion-spin theory has been proposed 25 , where the constrained electron operators are decoupled as, $C_{i a \uparrow}=h_{i a \uparrow}^{\dagger} S_{i a}^{-}$and $C_{i a \downarrow}=h_{i a \downarrow}^{\dagger} S_{i a}^{+}$, with the spinful fermion operator $h_{i a \sigma}=e^{-i \Phi_{i a \sigma}} h_{i a}$ represents the charge degree of freedom together with some effects of the spin configuration rearrangements due to the presence of the doped hole itself (dressed holon), while the spin operator $S_{i a}$ represents the spin degree of freedom, then the bilayer $t-t^{\prime}-J$ Hamiltonian (1) can be expressed in this CSS fermion-spin representation as,

$$
\begin{aligned}
H & =t \sum_{i \hat{\eta} a}\left(h_{i+\hat{\eta} a \uparrow}^{\dagger} h_{i a \uparrow} S_{i a}^{+} S_{i+\hat{\eta} a}^{-}+h_{i+\hat{\eta} a \downarrow}^{\dagger} h_{i a \downarrow} S_{i a}^{-} S_{i+\hat{\eta} a}^{+}\right) \\
& -t^{\prime} \sum_{i \hat{\tau} a}\left(h_{i+\hat{\tau} a \uparrow}^{\dagger} h_{i a \uparrow} S_{i a}^{+} S_{i+\hat{\tau} a}^{-}+h_{i+\hat{\tau} a \downarrow}^{\dagger} h_{i a \downarrow} S_{i a}^{-} S_{i+\hat{\tau} a}^{+}\right) \\
& +\sum_{i} t_{\perp}(i)\left(h_{i 2 \uparrow}^{\dagger} h_{i 1 \uparrow} S_{i 1}^{+} S_{i 2}^{-}+h_{i 1 \uparrow}^{\dagger} h_{i 2 \uparrow} S_{i 2}^{+} S_{i 1}^{-}\right. \\
& \left.+h_{i 2 \downarrow}^{\dagger} h_{i 1 \downarrow} S_{i 1}^{-} S_{i 2}^{+}+h_{i 1 \downarrow}^{\dagger} h_{i 2 \downarrow} S_{i 2}^{-} S_{i 1}^{+}\right)-\mu \sum_{i a \sigma} h_{i a \sigma}^{\dagger} h_{i a \sigma} \\
& +J_{\mathrm{eff}} \sum_{i \hat{\eta} a} \mathbf{S}_{i a} \cdot \mathbf{S}_{i+\hat{\eta} a}+J_{\mathrm{eff} \perp} \sum_{i} \mathbf{S}_{i 1} \cdot \mathbf{S}_{i 2},
\end{aligned}
$$

where $J_{\text {eff }}=J(1-\delta)^{2}, J_{\text {eff } \perp}=J_{\perp}(1-\delta)^{2}$, and $\delta=$ $\left\langle h_{i a \sigma}^{\dagger} h_{i a \sigma}\right\rangle=\left\langle h_{i a}^{\dagger} h_{i a}\right\rangle$ is the doping concentration. It has been shown that the electron single occupancy local constraint is satisfied in analytical calculations within this CSS fermion-spin theory, and the double spinful fermion occupancy are ruled out automatically 25 . Although in common sense $h_{i a \sigma}$ is not a real spinful fermion, it behaves like a spinful fermion ${ }^{25}$. As in the single layer case $\frac{18}{}$, the kinetic energy terms in the bilayer $t-t^{\prime}-J$ model have been transferred as the dressed holon-spin interactions, which can induce the dressed holon pairing state (hence the electron Cooper pairing state) by exchanging spin excitations in the higher power of the doping concentration. Before calculation of the electron normal and anomalous Green's functions of the bilayer system in the SC state, we firstly introduce the SC order parameter. As we have mentioned above, there are two coupled $\mathrm{CuO}_{2}$ planes in the unit cell, and in this case, the SC order parameters for the electron Cooper pair is a matrix $\Delta=\Delta_{L}+\sigma_{x} \Delta_{T}$, with the longitudinal and transverse SC order parameters in the CSS fermion-spin theory can be expressed as,

$$
\begin{aligned}
\Delta_{L} & =\left\langle C_{i a \uparrow}^{\dagger} C_{i+\hat{\eta} a \downarrow}^{\dagger}-C_{i a \downarrow}^{\dagger} C_{i+\hat{\eta} a \uparrow}^{\dagger}\right\rangle \\
& =\left\langle h_{i a \uparrow} h_{i+\hat{\eta} a \downarrow} S_{i a}^{+} S_{i+\hat{\eta} a}^{-}-h_{i a \downarrow} h_{i+\hat{\eta} a \uparrow} S_{i a}^{-} S_{i+\hat{\eta} a}^{+}\right\rangle \\
& =-\chi_{1} \Delta_{h L}, \\
\Delta_{T} & =\left\langle C_{i 1 \uparrow}^{\dagger} C_{i 2 \downarrow}^{\dagger}-C_{i 1 \downarrow}^{\dagger} C_{i 2 \uparrow}^{\dagger}\right\rangle \\
& =\left\langle h_{i 1 \uparrow} h_{i 2 \downarrow} S_{i 1}^{+} S_{i 2}^{-}-h_{i 1 \downarrow} h_{i 2 \uparrow} S_{i 1}^{-} S_{i 2}^{+}\right\rangle \\
& =-\chi_{\perp} \Delta_{h T},
\end{aligned}
$$


respectively, where the spin correlation functions $\chi_{1}=$ $\left\langle S_{i a}^{+} S_{i+\hat{\eta} a}^{-}\right\rangle$and $\chi_{\perp}=\left\langle S_{i 1}^{+} S_{i 2}^{-}\right\rangle$, and the longitudinal and transverse dressed holon pairing order parameters $\Delta_{h L}=\left\langle h_{i+\hat{\eta} a \downarrow} h_{i a \uparrow}-h_{i+\hat{\eta} a \uparrow} h_{i a \downarrow}\right\rangle$ and $\Delta_{h T}=\left\langle h_{i 2 \downarrow} h_{i 1 \uparrow}-\right.$ $\left.h_{i 2 \uparrow} h_{i 1 \downarrow}\right\rangle$.

Within the $t-J$ type model, robust indications of superconductivity with the d-wave symmetry in doped cuprates have been found by using numerical techniques ${ }^{26}$. On the other hand, it has been argued that the SC transition in doped cuprates is determined by the need to reduce the frustrated kinetic energy 27 . Although it is not necessary for the strong coupling of the electron quasiparticles and a pairing boson in their arguments ${ }^{27}$, a series of the inelastic neutron scattering experimental results provide a clear link between the electron quasiparticles and magnetic excitations ${ }^{28,29}$. In particular, an impurity-substitution effect on the low energy dynamics has been studied by virtue of the ARPES measurement ${ }^{30}$, this impurity-substitution effect is a magnetic analogue of the isotope effect used for the conventional superconductors. These experimental results ${ }^{30}$ reveal that the impurity-induced changes in the electron self-energy show a good correspondence to those of the magnetic excitations, indicating the importance of the magnetic fluctuation to the electron pairing in cuprate supercon- ductors. Recently, we ${ }^{19}$ have developed the kinetic energy driven SC mechanism based on the CSS fermionspin theory $\stackrel{25}{ }$, where the dressed holons interact occurring directly through the kinetic energy by exchanging spin excitations, leading to a net attractive force between dressed holons, then the electron Cooper pairs originating from the dressed holon pairing state are due to the charge-spin recombination, and their condensation reveals the $\mathrm{SC}$ ground-state. Within this $\mathrm{SC}$ mechanism ${ }^{19}$, the doping and temperature dependence of the electron spectral function of the single layer cuprate superconductors in the SC state has been discussed 18 . In this section, our main goal is to generalize these analytical calculations from the single layer case to the bilayer system. As in the case for the SC order parameter, the full dressed holon normal and anomalous Green's functions can also be expressed as $g(\mathbf{k}, \omega)=g_{L}(\mathbf{k}, \omega)+\sigma_{x} g_{T}(\mathbf{k}, \omega)$ and $\Im^{\dagger}(\mathbf{k}, \omega)=\Im_{L}^{\dagger}(\mathbf{k}, \omega)+\sigma_{x} \Im_{L}^{\dagger}(\mathbf{k}, \omega)$, respectively. We now can follow the previous discussions for the single layer case $\mathrm{e}^{18,19}$, and evaluate explicitly these corresponding longitudinal and transverse parts of the full dressed holon normal and anomalous Green's functions as [see the Appendix],

$$
\begin{aligned}
g_{L}(\mathbf{k}, \omega) & =\frac{1}{2} \sum_{\nu=1,2} Z_{h F A}^{(\nu)}\left(\frac{U_{h \nu \mathbf{k}}^{2}}{\omega-E_{h \nu \mathbf{k}}}+\frac{V_{h \nu \mathbf{k}}^{2}}{\omega+E_{h \nu \mathbf{k}}}\right), \\
g_{T}(\mathbf{k}, \omega) & =\frac{1}{2} \sum_{\nu=1,2}(-1)^{\nu+1} Z_{h F A}^{(\nu)}\left(\frac{U_{h \nu \mathbf{k}}^{2}}{\omega-E_{h \nu \mathbf{k}}}+\frac{V_{h \nu \mathbf{k}}^{2}}{\omega+E_{h \nu \mathbf{k}}}\right), \\
\Im_{L}^{\dagger}(\mathbf{k}, \omega) & =-\frac{1}{2} \sum_{\nu=1,2} Z_{h F A}^{(\nu)} \frac{\bar{\Delta}_{h z}^{(\nu)}(\mathbf{k})}{2 E_{h \nu \mathbf{k}}}\left(\frac{1}{\omega-E_{h \nu \mathbf{k}}}-\frac{1}{\omega+E_{h \nu \mathbf{k}}}\right), \\
\Im_{T}^{\dagger}(\mathbf{k}, \omega) & =-\frac{1}{2} \sum_{\nu=1,2}(-1)^{\nu+1} Z_{h F A}^{(\nu)} \frac{\bar{\Delta}_{h z}^{(\nu)}(\mathbf{k})}{2 E_{h \nu \mathbf{k}}}\left(\frac{1}{\omega-E_{h \nu \mathbf{k}}}-\frac{1}{\omega+E_{h \nu \mathbf{k}}}\right),
\end{aligned}
$$

where the dressed holon quasiparticle coherence factors $U_{h \nu \mathbf{k}}^{2}=\left[1+\bar{\xi}_{\nu \mathbf{k}} / E_{h \nu \mathbf{k}}\right] / 2$ and $V_{h \nu \mathbf{k}}^{2}=[1-$ $\left.\bar{\xi}_{\nu \mathbf{k}} / E_{h \nu \mathbf{k}}\right] / 2$, the dressed holon quasiparticle dispersion $E_{h \nu \mathbf{k}}=\sqrt{\left[\bar{\xi}_{\nu \mathbf{k}}\right]^{2}+\left|\bar{\Delta}_{h z}^{(\nu)}(\mathbf{k})\right|^{2}}$, the renormalized dressed holon excitation spectrum $\bar{\xi}_{\nu \mathbf{k}}=Z_{h F A}^{(\nu)} \xi_{\nu \mathbf{k}}$, with the mean-field (MF) dressed holon excitation spectrum $\xi_{\nu \mathbf{k}}=Z t \chi_{1} \gamma_{\mathbf{k}}-Z t^{\prime} \chi_{2} \gamma_{\mathbf{k}}^{\prime}-\mu+(-1)^{\nu+1} \chi_{\perp} t_{\perp}(\mathbf{k})$, where the spin correlation function $\chi_{2}=\left\langle S_{i a}^{+} S_{i+\hat{\tau} a}^{-}\right\rangle, \gamma_{\mathbf{k}}=$ $(1 / Z) \sum_{\hat{\eta}} e^{i \mathbf{k} \cdot \hat{\eta}}, \gamma_{\mathbf{k}}^{\prime}=(1 / Z) \sum_{\hat{\tau}} e^{i \mathbf{k} \cdot \hat{\tau}}, Z$ is the number of the nearest neighbor or next nearest neighbor sites, the renormalized dressed holon pair gap function $\bar{\Delta}_{h z}^{(\nu)}(\mathbf{k})=Z_{h F A}^{(\nu)}\left[\bar{\Delta}_{h L}(\mathbf{k})+(-1)^{\nu+1} \bar{\Delta}_{h T}(\mathbf{k})\right]$, with $\nu=1(\nu=2)$ for the bonding (antibonding) case, where $\bar{\Delta}_{h L}(\mathbf{k})=\left.\Sigma_{2 L}^{(h)}(\mathbf{k}, \omega)\right|_{\omega=0}=\bar{\Delta}_{h L} \gamma_{\mathbf{k}}^{(d)}$, with $\gamma_{\mathbf{k}}^{(d)}=$ $\left(\cos k_{x}-\cos k_{y}\right) / 2, \bar{\Delta}_{h T}(\mathbf{k})=\left.\Sigma_{2 T}^{(h)}(\mathbf{k}, \omega)\right|_{\omega=0}=\bar{\Delta}_{h T}$, the dressed holon quasiparticle coherent weights $Z_{h F A}^{(1)-1}=$ $Z_{h F 1}^{-1}-Z_{h F 2}^{-1}, \quad Z_{h F A}^{(2)-1}=Z_{h F 1}^{-1}+Z_{h F 2}^{-1}$, with $Z_{h F 1}^{-1}=$ $1-\left.\Sigma_{1 L}^{(h o)}\left(\mathbf{k}_{0}, \omega\right)\right|_{\omega=0}$, and $Z_{h F 2}^{-1}=\left.\Sigma_{1 T}^{(h o)}\left(\mathbf{k}_{0}, \omega\right)\right|_{\omega=0}$, where $\mathbf{k}_{0}=[\pi, 0], \Sigma_{1 L}^{(h o)}(\mathbf{k}, \omega)$ and $\Sigma_{1 T}^{(h o)}(\mathbf{k}, \omega)$ are the corresponding antisymmetric parts of the longitudinal and transverse dressed holon self-energy functions $\Sigma_{1 L}^{(h)}(\mathbf{k}, \omega)$ and $\Sigma_{1 T}^{(h)}(\mathbf{k}, \omega)$, while the longitudinal and transverse parts of the dressed holon self-energy functions $\Sigma_{1}^{(h)}(\mathbf{k}, \omega)$ and $\Sigma_{2}^{(h)}(\mathbf{k}, \omega)$ have been evaluated as, 


$$
\begin{aligned}
\Sigma_{1 L}^{(h)}\left(\mathbf{k}, i \omega_{n}\right) & =\frac{1}{N^{2}} \sum_{\mathbf{p}, \mathbf{q}}\left[R_{\mathbf{p}+\mathbf{q}+\mathbf{k}}^{(1)} \frac{1}{\beta} \sum_{i p_{m}} g_{L}\left(\mathbf{p}+\mathbf{k}, i p_{m}+i \omega_{n}\right) \Pi_{L L}\left(\mathbf{p}, \mathbf{q}, i p_{m}\right)\right. \\
& \left.+R_{\mathbf{p}+\mathbf{q}+\mathbf{k}}^{(2)} \frac{1}{\beta} \sum_{i p_{m}} g_{T}\left(\mathbf{p}+\mathbf{k}, i p_{m}+i \omega_{n}\right) \Pi_{T L}\left(\mathbf{p}, \mathbf{q}, i p_{m}\right)\right], \\
\Sigma_{1 T}^{(h)}\left(\mathbf{k}, i \omega_{n}\right) & =\frac{1}{N^{2}} \sum_{\mathbf{p}, \mathbf{q}}\left[R_{\mathbf{p}+\mathbf{q}+\mathbf{k}}^{(1)} \frac{1}{\beta} \sum_{i p_{m}} g_{T}\left(\mathbf{p}+\mathbf{k}, i p_{m}+i \omega_{n}\right) \Pi_{T T}\left(\mathbf{p}, \mathbf{q}, i p_{m}\right)\right. \\
& \left.+R_{\mathbf{p}+\mathbf{q}+\mathbf{k}}^{(2)} \frac{1}{\beta} \sum_{i p_{m}} g_{L}\left(\mathbf{p}+\mathbf{k}, i p_{m}+i \omega_{n}\right) \Pi_{L T}\left(\mathbf{p}, \mathbf{q}, i p_{m}\right)\right], \\
\Sigma_{2 L}^{(h)}\left(\mathbf{k}, i \omega_{n}\right) & =\frac{1}{N^{2}} \sum_{\mathbf{p}, \mathbf{q}}\left[R_{\mathbf{p}+\mathbf{q}+\mathbf{k}}^{(1)} \frac{1}{\beta} \sum_{i p_{m}} \Im_{L}^{\dagger}\left(-\mathbf{p}-\mathbf{k},-i p_{m}-i \omega_{n}\right) \Pi_{L L}\left(\mathbf{p}, \mathbf{q}, i p_{m}\right)\right. \\
& \left.+R_{\mathbf{p}+\mathbf{q}+\mathbf{k}}^{(2)} \frac{1}{\beta} \sum_{i p_{m}} \Im_{T}^{\dagger}\left(-\mathbf{p}-\mathbf{k},-i p_{m}-i \omega_{n}\right) \Pi_{T L}\left(\mathbf{p}, \mathbf{q}, i p_{m}\right)\right], \\
\Sigma_{2 T}^{(h)}\left(\mathbf{k}, i \omega_{n}\right) & =\frac{1}{N^{2}} \sum_{\mathbf{p}, \mathbf{q}}\left[R_{\mathbf{p}+\mathbf{q}+\mathbf{k}}^{(1)} \frac{1}{\beta} \sum_{i p_{m}} \Im_{T}^{\dagger}\left(-\mathbf{p}-\mathbf{k},-i p_{m}-i \omega_{n}\right) \Pi_{T T}\left(\mathbf{p}, \mathbf{q}, i p_{m}\right)\right. \\
& \left.+R_{\mathbf{p}+\mathbf{q}+\mathbf{k}}^{(2)} \frac{1}{\beta} \sum_{i p_{m}} \Im_{L}^{\dagger}\left(-\mathbf{p}-\mathbf{k},-i p_{m}-i \omega_{n}\right) \Pi_{L T}\left(\mathbf{p}, \mathbf{q}, i p_{m}\right)\right],
\end{aligned}
$$

where $R_{\mathbf{k}}^{(1)}=\left[Z\left(t \gamma_{\mathbf{k}}-t^{\prime} \gamma_{\mathbf{k}}^{\prime}\right)\right]^{2}+t_{\perp}^{2}(\mathbf{k}), R_{\mathbf{k}}^{(2)}=2 Z\left(t \gamma_{\mathbf{k}}-\right.$ $\left.t^{\prime} \gamma_{\mathbf{k}}^{\prime}\right) t_{\perp}(\mathbf{k})$, and the spin bubbles $\Pi_{\eta, \eta^{\prime}}\left(\mathbf{p}, \mathbf{q}, i p_{m}\right)=$ $(1 / \beta) \sum_{i q_{m}} D_{\eta}^{(0)}\left(\mathbf{q}, i q_{m}\right) D_{\eta^{\prime}}^{(0)}\left(\mathbf{q}+\mathbf{p}, i q_{m}+i p_{m}\right)$, with $\eta=$ $L, T$ and $\eta^{\prime}=L, T$, and the MF spin Green's function $D^{(0)}(\mathbf{k}, \omega)=D_{L}^{(0)}(\mathbf{k}, \omega)+\sigma_{x} D_{T}^{(0)}(\mathbf{k}, \omega)$, with the corresponding longitudinal and transverse parts have been given by $\stackrel{22}{ }$,

$$
\begin{aligned}
D_{L}^{(0)}(\mathbf{k}, \omega) & =\frac{1}{2} \sum_{\nu=1,2} \frac{B_{\nu \mathbf{k}}}{\omega^{2}-\omega_{\nu \mathbf{k}}^{2}}, \\
D_{T}^{(0)}(\mathbf{k}, \omega) & =\frac{1}{2} \sum_{\nu=1,2}(-1)^{\nu+1} \frac{B_{\nu \mathbf{k}}}{\omega^{2}-\omega_{\nu \mathbf{k}}^{2}},
\end{aligned}
$$

where $B_{\nu \mathbf{k}}=\lambda\left(A_{1} \gamma_{\mathbf{k}}-A_{2}\right)-\lambda^{\prime}\left(2 \chi_{2}^{z} \gamma_{\mathbf{k}}^{\prime}-\chi_{2}\right)-J_{\text {eff } \perp}\left[\chi_{\perp}+\right.$ $\left.2 \chi_{\perp}^{z}(-1)^{\nu}\right]\left[\epsilon_{\perp}(\mathbf{k})+(-1)^{\nu}\right], A_{1}=2 \epsilon_{\|} \chi_{1}^{z}+\chi_{1}, A_{2}=\epsilon_{\|} \chi_{1}+$ $2 \chi_{1}^{z}, \lambda=2 Z J_{\mathrm{eff}}, \lambda^{\prime}=4 Z \phi_{2} t^{\prime}, \epsilon_{\|}=1+2 t \phi_{1} / J_{\mathrm{eff}}, \epsilon_{\perp}(\mathbf{k})=$ $1+4 \phi_{\perp} t_{\perp}(\mathbf{k}) / J_{\text {eff } \perp}$, the spin correlation functions $\chi_{1}^{z}=$ $\left\langle S_{i a}^{z} S_{i+\hat{\eta} a}^{z}\right\rangle, \chi_{2}^{z}=\left\langle S_{i a}^{z} S_{i+\hat{\tau} a}^{z}\right\rangle, \chi_{\perp}^{z}=\left\langle S_{i 1}^{z} S_{i 2}^{z}\right\rangle$, the dressed holon particle-hole order parameters $\phi_{1}=\left\langle h_{i a \sigma}^{\dagger} h_{i+\eta \hat{n} a \sigma}\right\rangle$, $\phi_{2}=\left\langle h_{i a \sigma}^{\dagger} h_{i+\hat{\tau} a \sigma}\right\rangle, \phi_{\perp}=\left\langle h_{i 1 \sigma}^{\dagger} h_{i 2 \sigma}\right\rangle$, and the MF spin excitation spectrum,

$$
\begin{aligned}
\omega_{\nu \mathbf{k}}^{2} & =\lambda^{2}\left[\left(A_{4}-\alpha \epsilon_{\|} \chi_{1}^{z} \gamma_{\mathbf{k}}-\frac{1}{2 Z} \alpha \epsilon_{\|} \chi_{1}\right)\left(1-\epsilon_{\|} \gamma_{\mathbf{k}}\right)+\frac{1}{2} \epsilon_{\|}\left(A_{3}-\frac{2}{Z} \alpha \chi_{1}^{z}-\alpha \chi_{1} \gamma_{\mathbf{k}}\right)\left(\epsilon_{\|}-\gamma_{\mathbf{k}}\right)\right] \\
& +\lambda^{\prime 2}\left[\alpha\left(\chi_{2}^{z} \gamma_{\mathbf{k}}^{\prime}-\frac{Z-1}{2 Z} \chi_{2}\right) \gamma_{\mathbf{k}}^{\prime}+\frac{1}{2}\left(A_{5}-\frac{2}{Z} \alpha \chi_{2}^{z}\right)\right]+\lambda \lambda^{\prime} \alpha\left[\chi_{1}^{z}\left(1-\epsilon_{\|} \gamma_{\mathbf{k}}\right) \gamma_{\mathbf{k}}^{\prime}+\frac{1}{2}\left(\chi_{1} \gamma_{\mathbf{k}}^{\prime}-C_{2}\right)\left(\epsilon_{\|}-\gamma_{\mathbf{k}}\right)\right. \\
& \left.+\gamma_{\mathbf{k}}^{\prime}\left(C_{2}^{z}-\epsilon_{\|} \chi_{2}^{z} \gamma_{\mathbf{k}}\right)-\frac{1}{2} \epsilon_{\|}\left(C_{2}-\chi_{2} \gamma_{\mathbf{k}}\right)\right]+\lambda J_{\mathrm{eff} \perp} \alpha\left\{\frac{1}{2} \epsilon_{\perp}(\mathbf{k})\left(\epsilon_{\|}-\gamma_{\mathbf{k}}\right)\left[C_{\perp}+\chi_{1}(-1)^{\nu}\right]\right. \\
& \left.+\left(1-\epsilon_{\|} \gamma_{\mathbf{k}}\right)\left[C_{\perp}^{z}+\chi_{1}^{z} \epsilon_{\perp}(\mathbf{k})(-1)^{\nu}\right]+\left[\epsilon_{\perp}(\mathbf{k})+(-1)^{\nu}\right]\left[\frac{1}{2} \epsilon_{\|}\left(C_{\perp}-\chi_{\perp} \gamma_{\mathbf{k}}\right)+\left(C_{\perp}^{z}-\epsilon_{\|} \chi_{\perp}^{z} \gamma_{\mathbf{k}}\right)(-1)^{\nu}\right]\right\} \\
& +\lambda^{\prime} J_{\mathrm{eff} \perp} \alpha\left\{\gamma_{\mathbf{k}}^{\prime}\left[C_{\perp}^{\prime z}+\chi_{2}^{z} \epsilon_{\perp}(\mathbf{k})(-1)^{\nu}\right]-\frac{1}{2} \epsilon_{\perp}(\mathbf{k})\left[C_{\perp}^{\prime}+\chi_{2}(-1)^{\nu}\right]\right. \\
& \left.+\left[\frac{1}{2}\left(\chi_{\perp} \gamma_{\mathbf{k}}^{\prime}-C_{\perp}^{\prime}\right)+\chi_{\perp}^{z} \gamma_{\mathbf{k}}^{\prime}(-1)^{\nu}\right]\left[\epsilon_{\perp}(\mathbf{k})+(-1)^{\nu}\right]\right\}+\frac{1}{4} J_{\mathrm{eff} \perp}^{2}\left[\epsilon_{\perp}(\mathbf{k})+(-1)^{\nu}\right]^{2}
\end{aligned}
$$

where $A_{3}=\alpha C_{1}+(1-\alpha) / 2 Z, A_{4}=\alpha C_{1}^{z}+(1-\alpha) / 4 Z, \quad$ tion functions $\quad C_{1} \quad=\quad\left(1 / Z^{2}\right) \sum_{\hat{\eta} \hat{\eta}^{\prime}}\left\langle S_{i+\hat{\eta} a}^{+} S_{i+\hat{\eta}^{\prime} a}^{-}\right\rangle$, $A_{5}=\alpha C_{3}+(1-\alpha) / 2 Z$, and the spin correla- 
$C_{2}=\left(1 / Z^{2}\right) \sum_{\hat{\eta} \hat{\tau}}\left\langle S_{i+\hat{\eta} a}^{+} S_{i+\hat{\tau} a}^{-}\right\rangle, \quad C_{3}=$ $\left(1 / Z^{2}\right) \sum_{\hat{\hat{\tau}} \hat{\tau}^{\prime}}\left\langle S_{i+\hat{\tau} a}^{+} S_{i+\hat{\tau}^{\prime} a}^{-}\right\rangle, \quad \quad C_{1}^{z}=$ $\left(1 / Z^{2}\right) \sum_{\hat{\eta} \hat{\eta}^{\prime}}\left\langle S_{i+\hat{\eta} a}^{z} S_{i+\hat{\eta}^{\prime} a}^{z}\right\rangle, \quad C_{2}^{z}=$ $\left(1 / Z^{2}\right) \sum_{\hat{\eta} \hat{\tau}}\left\langle S_{i+\hat{\eta} a}^{z} S_{i+\hat{\tau} a}^{z}\right\rangle, C_{\perp}=(1 / Z) \sum_{\hat{\eta}}\left\langle S_{i 1}^{+} S_{i+\hat{\eta} 2}^{-}\right\rangle$, $C^{\prime}{ }_{\perp}=(1 / Z) \sum_{\hat{\tau}}\left\langle S_{i 1}^{+} S_{i+\hat{\tau} 2}^{-}\right\rangle, C_{\perp}^{z}=(1 / Z) \sum_{\hat{\eta}}\left\langle S_{i 1}^{z} S_{i+\hat{\eta} 2}^{z}\right\rangle$, and $C^{\prime z}=(1 / Z) \sum_{\hat{\tau}}\left\langle S_{i 1}^{z} S_{i+\hat{\tau} 2}^{z}\right\rangle$. In order to satisfy the sum rule of the spin correlation function $\left\langle S_{i a}^{+} S_{i a}^{-}\right\rangle=1 / 2$ in the case without AFLRO, the important decoupling parameter $\alpha$ has been introduced in the above calculation as in the single layer case ${ }^{18,19,22}$, which can be regarded as the vertex correction.

With the help of the longitudinal and transverse parts of the full dressed holon normal and anomalous Green's functions in Eq. (5) and MF spin Green's function in Eq. (7), we now can calculate the electron normal and anomalous Green's functions $G\left(i-j, t-t^{\prime}\right)=$ $\left\langle\left\langle C_{i \sigma}(t) ; C_{j \sigma}^{\dagger}\left(t^{\prime}\right)\right\rangle\right\rangle=G_{L}\left(i-j, t-t^{\prime}\right)+\sigma_{x} G_{T}\left(i-j, t-t^{\prime}\right)$ and $\Gamma^{\dagger}\left(i-j, t-t^{\prime}\right)=\left\langle\left\langle C_{i \uparrow}^{\dagger}(t) ; C_{j \downarrow}^{\dagger}\left(t^{\prime}\right)\right\rangle\right\rangle=\Gamma_{L}^{\dagger}\left(i-j, t-t^{\prime}\right)+$ $\sigma_{x} \Gamma_{T}^{\dagger}\left(i-j, t-t^{\prime}\right)$, where these longitudinal and transverse parts are the convolutions of the corresponding longitudinal and transverse parts of the full dressed holon normal and anomalous Green's functions and MF spin Green's function in the CSS fermion-spin theory, and can be evaluated explicitly as,

$$
\begin{aligned}
& G_{L}(\mathbf{k}, \omega)=\frac{1}{8 N} \sum_{\mathbf{p}} \sum_{\mu \nu} Z_{h F A}^{(\mu)} \frac{B_{\nu \mathbf{p}}}{\omega_{\nu \mathbf{p}}}\left[L_{\mu \nu}^{(1)}(\mathbf{k}, \mathbf{p})\left(\frac{U_{h \mu \mathbf{p}-\mathbf{k}}^{2}}{\omega+E_{h \mu \mathbf{p}-\mathbf{k}}-\omega_{\nu \mathbf{p}}}+\frac{V_{h \mu \mathbf{p}-\mathbf{k}}^{2}}{\omega-E_{h \mu \mathbf{p}-\mathbf{k}}+\omega_{\nu \mathbf{p}}}\right)\right. \\
& \left.+L_{\mu \nu}^{(2)}(\mathbf{k}, \mathbf{p})\left(\frac{U_{h \mu \mathbf{p}-\mathbf{k}}^{2}}{\omega+E_{h \mu \mathbf{p}-\mathbf{k}}+\omega_{\nu \mathbf{p}}}+\frac{V_{h \mu \mathbf{p}-\mathbf{k}}^{2}}{\omega-E_{h \mu \mathbf{p}-\mathbf{k}}-\omega_{\nu \mathbf{p}}}\right)\right], \\
& G_{T}(\mathbf{k}, \omega)=\frac{1}{8 N} \sum_{\mathbf{p}} \sum_{\mu \nu}(-1)^{\mu+\nu} Z_{h F A}^{(\mu)} \frac{B_{\nu \mathbf{p}}}{\omega_{\nu \mathbf{p}}}\left[L_{\mu \nu}^{(1)}(\mathbf{k}, \mathbf{p})\left(\frac{U_{h \mu \mathbf{p}-\mathbf{k}}^{2}}{\omega+E_{h \mu \mathbf{p}-\mathbf{k}}-\omega_{\nu \mathbf{p}}}+\frac{V_{h \mu \mathbf{p}-\mathbf{k}}^{2}}{\omega-E_{h \mu \mathbf{p}-\mathbf{k}}+\omega_{\nu \mathbf{p}}}\right)\right. \\
& \left.+L_{\mu \nu}^{(2)}(\mathbf{k}, \mathbf{p})\left(\frac{U_{h \mu \mathbf{p}-\mathbf{k}}^{2}}{\omega+E_{h \mu \mathbf{p}-\mathbf{k}}+\omega_{\nu \mathbf{p}}}+\frac{V_{h \mu \mathbf{p}-\mathbf{k}}^{2}}{\omega-E_{h \mu \mathbf{p}-\mathbf{k}}-\omega_{\nu \mathbf{p}}}\right)\right], \\
& \Gamma_{L}^{\dagger}(\mathbf{k}, \omega)=\frac{1}{8 N} \sum_{\mathbf{p}} \sum_{\mu \nu} Z_{h F A}^{(\mu)} \frac{\bar{\Delta}_{h z}^{(\mu)}(\mathbf{p}-\mathbf{k})}{2 E_{h \mu \mathbf{p}-\mathbf{k}}} \frac{B_{\nu \mathbf{p}}}{\omega_{\nu \mathbf{p}}}\left[L _ { \mu \nu } ^ { ( 1 ) } ( \mathbf { k } , \mathbf { p } ) \left(\frac{1}{\omega-E_{h \mu \mathbf{p}-\mathbf{k}}+\omega_{\nu \mathbf{p}}}\right.\right. \\
& \left.\left.-\frac{1}{\omega+E_{h \mu \mathbf{p}-\mathbf{k}}-\omega_{\nu \mathbf{p}}}\right)+L_{\mu \nu}^{(2)}(\mathbf{k}, \mathbf{p})\left(\frac{1}{\omega-E_{h \mu \mathbf{p}-\mathbf{k}}-\omega_{\nu \mathbf{p}}}-\frac{1}{\omega+E_{h \mu \mathbf{p}-\mathbf{k}}+\omega_{\nu \mathbf{p}}}\right)\right], \\
& \Gamma_{T}^{\dagger}(\mathbf{k}, \omega)=\frac{1}{8 N} \sum_{\mathbf{p}} \sum_{\mu \nu}(-1)^{\mu+\nu} Z_{h F A}^{(\mu)} \frac{\bar{\Delta}_{h z}^{(\mu)}(\mathbf{p}-\mathbf{k})}{2 E_{h \mu \mathbf{p}-\mathbf{k}}} \frac{B_{\nu \mathbf{p}}}{\omega_{\nu \mathbf{p}}}\left[L _ { \mu \nu } ^ { ( 1 ) } ( \mathbf { k } , \mathbf { p } ) \left(\frac{1}{\omega-E_{h \mu \mathbf{p}-\mathbf{k}}+\omega_{\nu \mathbf{p}}}\right.\right. \\
& \left.\left.-\frac{1}{\omega+E_{h \mu \mathbf{p}-\mathbf{k}}-\omega_{\nu \mathbf{p}}}\right)+L_{\mu \nu}^{(2)}(\mathbf{k}, \mathbf{p})\left(\frac{1}{\omega-E_{h \mu \mathbf{p}-\mathbf{k}}-\omega_{\nu \mathbf{p}}}-\frac{1}{\omega+E_{h \mu \mathbf{p}-\mathbf{k}}+\omega_{\nu \mathbf{p}}}\right)\right],
\end{aligned}
$$

where $L_{\mu \nu}^{(1)}(\mathbf{k}, \mathbf{p})=\left[\operatorname{coth}\left(\beta \omega_{\nu \mathbf{p}} / 2\right)-\operatorname{th}\left(\beta E_{h \mu \mathbf{p}-\mathbf{k}} / 2\right)\right] / 2$ and $L_{\mu \nu}^{(2)}(\mathbf{k}, \mathbf{p})=\left[\operatorname{coth}\left(\beta \omega_{\nu \mathbf{p}} / 2\right)+\operatorname{th}\left(\beta E_{h \mu \mathbf{p}-\mathbf{k}} / 2\right)\right] / 2$, then the longitudinal and transverse parts of the electron spectral function $A_{L}(\mathbf{k}, \omega)=-2 \operatorname{Im} G_{L}(\mathbf{k}, \omega)$ and $A_{T}(\mathbf{k}, \omega)=-2 \operatorname{Im} G_{T}(\mathbf{k}, \omega)$ and SC gap function $\Delta_{L}(\mathbf{k})=(1 / \beta) \sum_{i \omega_{n}} \Gamma_{L}^{\dagger}\left(\mathbf{k}, i \omega_{n}\right)$ and $\Delta_{T}(\mathbf{k})=$ $(1 / \beta) \sum_{i \omega_{n}} \Gamma_{T}^{\dagger}\left(\mathbf{k}, i \omega_{n}\right)$ are obtained as,

$$
\begin{aligned}
A_{L}(\mathbf{k}, \omega) & =\pi \frac{1}{4 N} \sum_{\mathbf{p}} \sum_{\mu \nu} Z_{h F A}^{(\mu)} \frac{B_{\nu \mathbf{p}}}{\omega_{\nu \mathbf{p}}}\left\{L_{\mu \nu}^{(1)}(\mathbf{k}, \mathbf{p})\left[U_{h \mu \mathbf{p}-\mathbf{k}}^{2} \delta\left(\omega+E_{h \mu \mathbf{p}-\mathbf{k}}-\omega_{\nu \mathbf{p}}\right)+V_{h \mu \mathbf{p}-\mathbf{k}}^{2} \delta\left(\omega-E_{h \mu \mathbf{p}-\mathbf{k}}+\omega_{\nu \mathbf{p}}\right)\right]\right. \\
& \left.+L_{\mu \nu}^{(2)}(\mathbf{k}, \mathbf{p})\left[U_{h \mu \mathbf{p}-\mathbf{k}}^{2} \delta\left(\omega+E_{h \mu \mathbf{p}-\mathbf{k}}+\omega_{\nu \mathbf{p}}\right)+V_{h \mu \mathbf{p}-\mathbf{k}}^{2} \delta\left(\omega-E_{h \mu \mathbf{p}-\mathbf{k}}-\omega_{\nu \mathbf{p}}\right)\right]\right\} \\
A_{T}(\mathbf{k}, \omega) & =\pi \frac{1}{4 N} \sum_{\mathbf{p}} \sum_{\mu \nu}(-1)^{\mu+\nu} Z_{h F A}^{(\mu)} \frac{B_{\nu \mathbf{p}}}{\omega_{\nu \mathbf{p}}}\left\{L_{\mu \nu}^{(1)}(\mathbf{k}, \mathbf{p})\left[U_{h \mu \mathbf{p}-\mathbf{k}}^{2} \delta\left(\omega+E_{h \mu \mathbf{p}-\mathbf{k}}-\omega_{\nu \mathbf{p}}\right)+V_{h \mu \mathbf{p}-\mathbf{k}}^{2} \delta\left(\omega-E_{h \mu \mathbf{p}-\mathbf{k}}+\omega_{\nu \mathbf{p}}\right)\right]\right. \\
& \left.+L_{\mu \nu}^{(2)}(\mathbf{k}, \mathbf{p})\left[U_{h \mu \mathbf{p}-\mathbf{k}}^{2} \delta\left(\omega+E_{h \mu \mathbf{p}-\mathbf{k}}+\omega_{\nu \mathbf{p}}\right)+V_{h \mu \mathbf{p}-\mathbf{k}}^{2} \delta\left(\omega-E_{h \mu \mathbf{p}-\mathbf{k}}-\omega_{\nu \mathbf{p}}\right)\right]\right\}
\end{aligned}
$$




$$
\begin{aligned}
& \Delta_{L}(\mathbf{k})=-\frac{1}{16 N} \sum_{\mathbf{p}, \mu, \nu} Z_{h F A}^{(\mu)} \frac{\bar{\Delta}_{h z}^{(\mu)}(\mathbf{p}-\mathbf{k})}{E_{h \mu \mathbf{p}-\mathbf{k}}} \frac{B_{\nu \mathbf{p}}}{\omega_{\nu \mathbf{p}}} \operatorname{th}\left[\frac{1}{2} \beta E_{h \mu \mathbf{p}-\mathbf{k}}\right] \operatorname{coth}\left[\frac{1}{2} \beta \omega_{\nu \mathbf{p}}\right], \\
& \Delta_{T}(\mathbf{k})=-\frac{1}{16 N} \sum_{\mathbf{p}, \mu, \nu}(-1)^{\mu+\nu} Z_{h F A}^{(\mu)} \frac{\bar{\Delta}_{h z}^{(\mu)}(\mathbf{p}-\mathbf{k})}{E_{h \mu \mathbf{p}-\mathbf{k}}} \frac{B_{\nu \mathbf{p}}}{\omega_{\nu \mathbf{p}}} \operatorname{th}\left[\frac{1}{2} \beta E_{h \mu \mathbf{p}-\mathbf{k}}\right] \operatorname{coth}\left[\frac{1}{2} \beta \omega_{\nu \mathbf{p}}\right] .
\end{aligned}
$$

With the above longitudinal and transverse parts of the SC gap functions in Eqs. (10c) and (10d), the corresponding longitudinal and transverse SC gap parameters are obtained as $\Delta_{L}=-\chi_{1} \Delta_{h L}$ and $\Delta_{T}=-\chi_{\perp} \Delta_{h T}$, respectively. In the bilayer coupling case, the more appropriate classification is in terms of the spectral function and SC gap function within the basis of the antibonding and bonding components $13,14,15,16,17$. In this case, the electron spectral function and SC gap parameter can be transformed from the plane representation to the antibonding-bonding representation as,

$$
\begin{aligned}
A^{(a)}(\mathbf{k}, \omega) & =\frac{1}{2}\left[A_{L}(\mathbf{k}, \omega)-A_{T}(\mathbf{k}, \omega)\right], \\
A^{(b)}(\mathbf{k}, \omega) & =\frac{1}{2}\left[A_{L}(\mathbf{k}, \omega)+A_{T}(\mathbf{k}, \omega)\right], \\
\Delta^{(a)} & =\Delta_{L}-\Delta_{T}, \\
\Delta^{(b)} & =\Delta_{L}+\Delta_{T} .
\end{aligned}
$$

respectively, then the antibonding and bonding parts have odd and even symmetries, respectively.

\section{ELECTRON STRUCTURE OF BILAYER CUPRATE SUPERCONDUCTORS}

We now begin to discuss the effect of the bilayer interaction on the electronic structure in the SC state. We first plot, in Fig. 1, the antibonding (solid line) and bonding (dashed line) electron spectral functions in the $[\pi, 0]$ point for parameters $t / J=2.5, t^{\prime} / t=0.3$, and $t_{\perp} / t=0.35$ with temperature $T=0.002 J$ at the doping concentration $\delta=0.15$. In comparison with the single layer case ${ }^{18}$, the electron spectrum of the bilayer system has been split into the bonding and antibonging components, with the bonding and antibonding SC quasiparticle peaks in the $[\pi, 0]$ point are located at the different positions. In this sense, the differentiation between the bonding and antibonding components of the electron spectral function is essential. The antibonding spectrum consists of a low energy antibonding peak, corresponding to the SC peak, and the bonding spectrum has a higher energy bonding peak, corresponding to the hump, while the spectral dip is in between them, then the total contributions for the electron spectrum from both antibonding and bonding components give rise to the PDH structure. Although the simple bilayer $t-t^{\prime}-J$ model (1) cannot be regarded as a comprehensive model for a quantitative comparison with bilayer cuprate superconductors, our present results for the SC state are in qualitative agreement with the major experimental observations on bilayer cuprate superconductors $2,7,8,9,10,11,16$.

We now turn to discuss the doping evolution of the electron spectrum of bilayer cuprate superconductors in

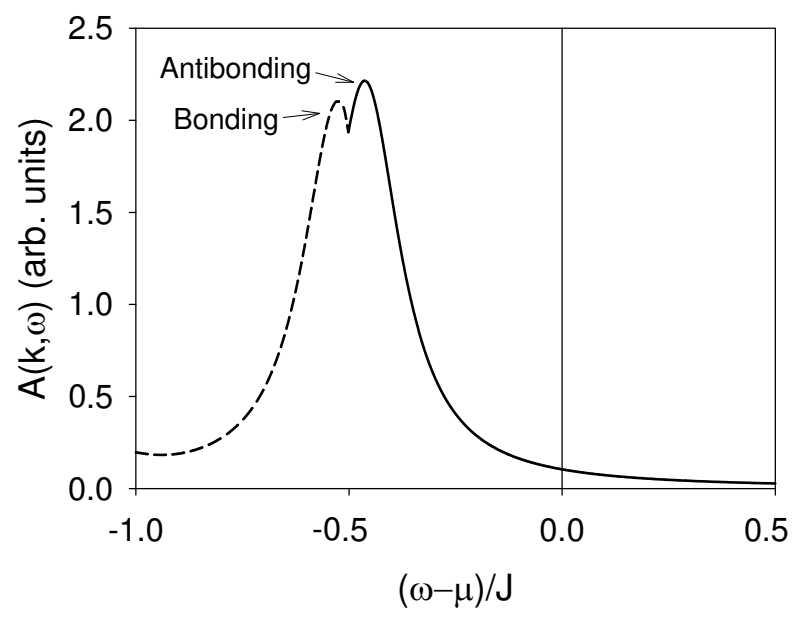

FIG. 1: The antibonding (solid line) and bonding (dashed line) electron spectral functions in the $[\pi, 0]$ point for $t / J=$ $2.5, t^{\prime} / t=0.3$, and $t_{\perp} / t=0.35$ with $T=0.002 J$ at $\delta=0.15$.

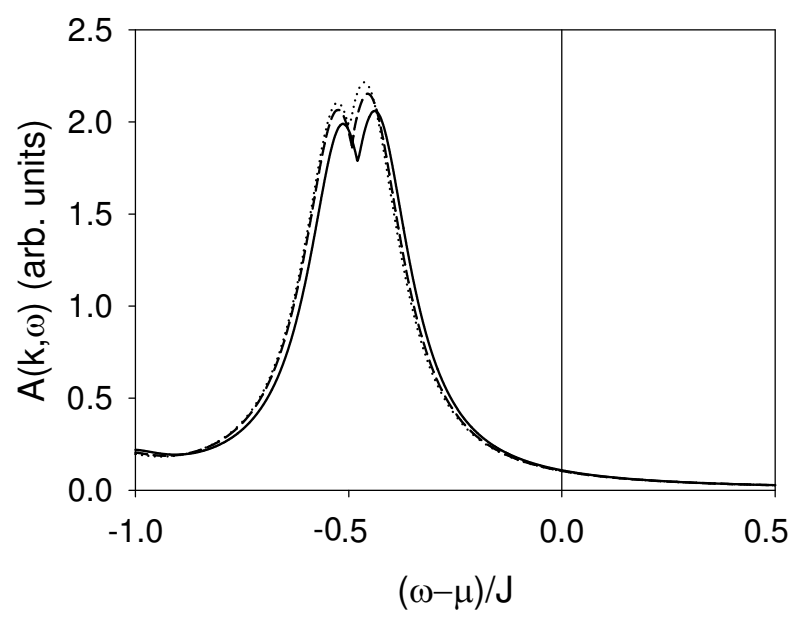

FIG. 2: The electron spectral functions at $[\pi, 0]$ point for $t / J=2.5, t^{\prime} / t=0.3$, and $t_{\perp} / t=0.35$ with $T=0.002 J$ at $\delta=0.09$ (solid line), $\delta=0.12$ (dashed line), and $\delta=0.15$ (dotted line).

the SC state. We have calculated the electron spectrum at different doping concentrations, and the result of the electron spectral functions in the $[\pi, 0]$ point for $t / J=2.5, t^{\prime} / t=0.3$, and $t_{\perp} / t=0.35$ with $T=0.002 J$ at $\delta=0.09$ (solid line), $\delta=0.12$ (dashed line), and $\delta=0.15$ (dotted line) are plotted in Fig. 2. In comparison with the corresponding ARPES experimental results of the bilayer cuprate superconductor $\mathrm{Bi}_{2} \mathrm{Sr}_{2} \mathrm{CaCu}_{2} \mathrm{O}_{8+\delta}$ in the SC state in Ref $\frac{12}{2}$, it is obviously that the doping evolution of the spectral weight of the bilayer supercon- 


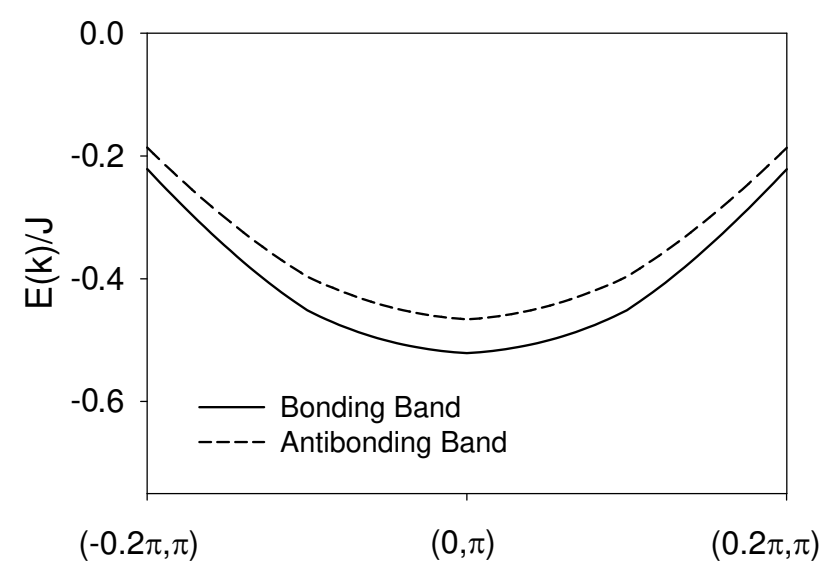

FIG. 3: The positions of the antibonding peaks and bonding humps in the electron spectrum as a function of momentum along the direction $[-0.2 \pi, \pi] \rightarrow[0, \pi] \rightarrow[0.2 \pi, \pi]$ with $T=$ $0.002 J$ at $\delta=0.15$ for $t / J=2.5, t^{\prime} / t=0.3$, and $t_{\perp} / t=0.35$.

ductor $\mathrm{Bi}_{2} \mathrm{Sr}_{2} \mathrm{CaCu}_{2} \mathrm{O}_{8+\delta}$ is reproduced. With increasing the doping concentration, both SC peak and hump become sharper, and then the spectral weights increase in intensity. Furthermore, we have also calculated the electron spectrum with different temperatures, and the results show that the spectral weights of both SC peak and hump are suppressed with increasing temperatures. Our these results are also qualitatively consistent with the ARPES experimental results on bilayer cuprate superconductors in the SC state $2,9,12$.

To better perceive the anomalous form of the antibonding and bonding electron spectral functions as a function of energy $\omega$ for $\mathbf{k}$ in the vicinity of the $[\pi, 0]$ point, we have made a series of calculations for the electron spectral function at different momenta, and the results show that the sharp SC peak from the electron antibonding spectral function and hump from the bonding spectral function persist in a very large momentum space region around the $[\pi, 0]$ point. To show this point clearly, we plot the positions of the antibonding peak and bonding hump in the electron spectrum as a function of momentum along the direction $[-0.2 \pi, \pi] \rightarrow[0, \pi] \rightarrow[0.2 \pi, \pi]$ with $T=0.002 J$ at $\delta=0.15$ for $t / J=2.5, t^{\prime} / t=0.3$, and $t_{\perp} / t=0.35$ in Fig. 3. Our result shows that there are two branches in the quasiparticle dispersion, with upper branch corresponding to the antibonding quasiparticle dispersion, and lower branch corresponding to the bonding quasiparticle dispersion. Furthermore, the BS reaches its maximum at the $[\pi, 0]$ point. Our present result also shows that in analogy to the two flat bands appeared in the normal state $\frac{22}{}$, both electron antibonding peak and bonding hump have a weak dispersion around the $[\pi, 0]$ point, in qualitative agreement with the ARPES experimental measurements on bilayer cuprate superconductors in the SC state $2,7,8,9,10,11,14$.

In the above calculations, we find that although the antibonding SC peak and bonding hump have different dispersions, the transverse part of the $\mathrm{SC}$ gap parameter $\Delta_{T} \approx 0$. To show this point clearly, we plot the antibonding and bonding gap parameters in Eqs. (11c)

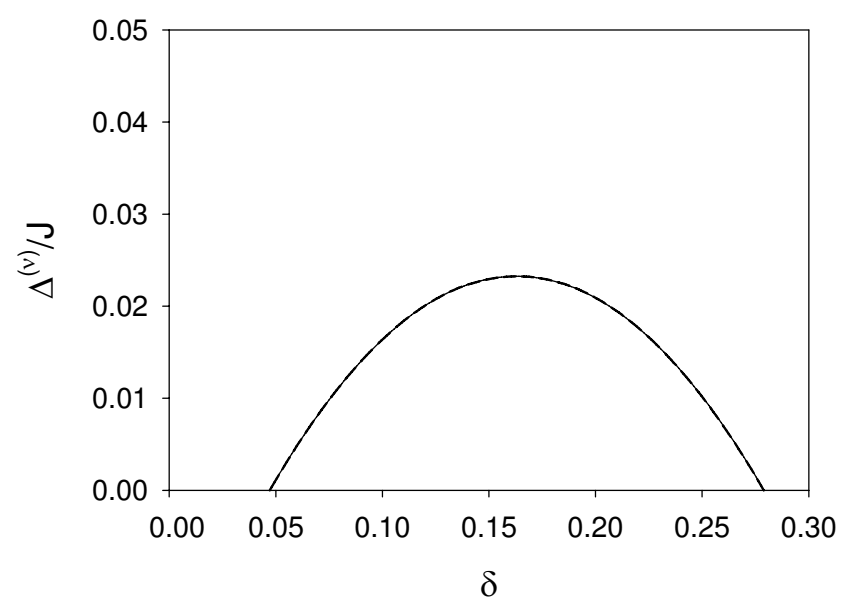

FIG. 4: The antibonding (solid line) and bonding (dashed line) gap parameters as a function of the doping concentration with $T=0.002 J$ for $t / J=2.5, t^{\prime} / t=0.3$, and $t_{\perp} / t=0.35$.

and (11d) as a function of the doping concentration with $T=0.002 J$ for $t / J=2.5, t^{\prime} / t=0.3$, and $t_{\perp} / t=0.35$ in Fig. 4. As seen from Fig. 4, both antibonding and bonding gap parameters have the same d-wave SC gap magnitude in a given doping concentration, i.e., $\Delta^{a} \approx \Delta^{b}$. This result shows that although there is a single electron interlayer coherent hopping (2) in bilayer cuprate superconductors in the SC state, the electron interlayer pairing interaction vanishes. This reflects that in the present kinetic energy driven SC mechanism, the weak dressed holon-spin interaction due to the interlayer coherent hopping (2) from the kinetic energy terms in Eq. (3) does not induce the dressed holon interlayer pairing state by exchanging spin excitations in the higher power of the doping concentration. This is different from the dressed holon-spin interaction due to the intralayer hopping from the kinetic energy terms in Eq. (3), it can induce superconductivity by exchanging spin excitations in the higher power of the doping concentration ${ }^{19}$. Our this result is also consistent with the ARPES experimental results of the bilayer cuprate superconductor $\mathrm{Bi}(\mathrm{Pb})_{2} \mathrm{Sr}_{2} \mathrm{CaCu}_{2} \mathrm{O}_{8+\delta}$ 14,16 , where the SC gap separately for the bonding and antibonding bands has been measured, and it is found that both d-wave SC gaps from the antibonding and bonding components are identical within the experimental uncertainties.

To our present understanding, two main reasons why the electronic structure of bilayer cuprate superconductors in the SC state can be described qualitatively in the framework of the kinetic energy driven superconductivity by considering the bilayer interaction are as follows. Firstly, the bilayer interaction causes the BS, this leads to that the full electron normal (anomalous) Green's function is divided into the longitudinal and transverse parts, respectively, then the bonding and antibonding electron spectral functions (SC gap functions) are obtained from these longitudinal and transverse parts of the electron normal (anomalous) Green's function, respectively. Although the transverse part of the SC gap parameter $\Delta_{T} \approx 0$, the antibonding peak around 
the $[\pi, 0]$ point is always at lower binding energy than the bonding peak (hump) due to the BS. In this sense, the PDH structure in the bilayer cuprate superconductors in the SC state is mainly caused by the BS. Secondly, the SC state in the kinetic energy driven SC mechanism is the conventional BCS like as in the single layer case $\mathrm{e}^{18.19}$. This can be understood from the electron normal and anomalous Green's functions in Eq. (9). Since the spins center around the $[\pi, \pi]$ point in the MF leve $1^{18,19,22}$, then the main contributions for the spins comes from the $[\pi, \pi]$ point. In this case, the longitudinal and transverse parts of the electron normal and anomalous Green's functions in Eq. (9) can be approximately reduced in terms of $\omega_{\nu \mathbf{p}=[\pi, \pi]} \sim 0$ and one of the self-consistent equations ${ }^{22} 1 / 2=\left\langle S_{i a}^{+} S_{i a}^{-}\right\rangle=$ $1 /(4 N) \sum_{\nu, \mathbf{k}}\left(B_{\nu \mathbf{k}} / \omega_{\nu \mathbf{k}}\right) \operatorname{coth}\left[(1 / 2) \beta \omega_{\nu \mathbf{k}}\right]$ as,

$G_{L}(\mathbf{k}, \omega) \approx \frac{1}{2} \sum_{\nu=1,2} Z_{F A}^{(\nu)}\left(\frac{U_{\nu \mathbf{k}}^{2}}{\omega-E_{\nu \mathbf{k}}}+\frac{V_{\nu \mathbf{k}}^{2}}{\omega+E_{\nu \mathbf{k}}}\right)$,

$G_{T}(\mathbf{k}, \omega) \approx \frac{1}{2} \sum_{\nu=1,2}(-1)^{\nu+1} Z_{F A}^{(\nu)}\left(\frac{U_{\nu \mathbf{k}}^{2}}{\omega-E_{\nu \mathbf{k}}}+\frac{V_{\nu \mathbf{k}}^{2}}{\omega+E_{\nu \mathbf{k}}}\right)$,

$\Gamma_{L}^{\dagger}(\mathbf{k}, \omega)=\frac{1}{2} \sum_{\nu=1,2} Z_{F A}^{(\nu)} \frac{\bar{\Delta}_{z}^{(\nu)}(\mathbf{k})}{2 E_{\nu \mathbf{k}}}\left(\frac{1}{\omega-E_{\nu \mathbf{k}}}-\frac{1}{\omega+E_{\nu \mathbf{k}}}\right)$

$$
\begin{aligned}
\Gamma_{T}^{\dagger}(\mathbf{k}, \omega)=\frac{1}{2} & \sum_{\nu=1,2}(-1)^{\nu+1} Z_{F A}^{(\nu)} \frac{\bar{\Delta}_{z}^{(\nu)}(\mathbf{k})}{2 E_{\nu \mathbf{k}}} \\
& \times\left(\frac{1}{\omega-E_{\nu \mathbf{k}}}-\frac{1}{\omega+E_{\nu \mathbf{k}}}\right),
\end{aligned}
$$

where the electron coherent weights $Z_{F A}^{(\nu)}=Z_{h F A}^{(\nu)} / 2$, the electron quasiparticle coherence factors $U_{\nu \mathbf{k}}^{2} \approx V_{h \nu \mathbf{k}-\mathbf{k}_{A}}^{2}$ and $V_{\nu \mathbf{k}}^{2} \approx U_{h \nu \mathbf{k}-\mathbf{k}_{A}}^{2}$, the SC gap function $\bar{\Delta}_{z}^{(\nu)}(\mathbf{k}) \approx$ $\bar{\Delta}_{h z}^{(\nu)}\left(\mathbf{k}-\mathbf{k}_{A}\right)$ and the electron quasiparticle spectrum $E_{\nu \mathbf{k}} \approx E_{h \nu \mathbf{k}-\mathbf{k}_{\mathbf{A}}}$, with $\mathbf{k}_{\mathbf{A}}=[\pi, \pi]$. As in the single layer case $\frac{18,19}{1}$, this reflects that the hole-like dressed holon quasiparticle coherence factors $V_{h \nu \mathbf{k}}$ and $U_{h \nu \mathbf{k}}$ and hole-like dressed holon quasiparticle spectrum $E_{h \nu \mathbf{k}}$ have been transferred into the electron quasiparticle coherence factors $U_{\nu \mathbf{k}}$ and $V_{\nu \mathbf{k}}$ and electron quasiparticle spectrum $E_{\nu \mathbf{k}}$, respectively, by the convolutions of the corresponding longitudinal and transverse parts of the MF spin Green's function and full dressed holon normal and anomalous Green's functions due to the charge-spin recombination 27 . As a result, these electron normal and anomalous Green's functions in Eq. (12) are typical bilayer BCS like $\underline{6}$. This also reflects that as in the single layer case $\frac{18,19}{1}$, the dressed holon pairs condense with the d-wave symmetry in a wide range of the doping concentration, then the electron Cooper pairs originating from the dressed holon pairing state are due to the chargespin recombination, and their condensation automatically gives the electron quasiparticle character. These are why the basic bilayer BCS formalism ${ }^{6}$ is still valid in discussions of SC coherence of the quasiparticle peak and hump, although the pairing mechanism is driven by the intralayer kinetic energy by exchanging spin excitations, and other exotic magnetic scattering 28,29 is beyond the BCS formalism.

\section{SUMMARY AND DISCUSSIONS}

We have studied the electronic structure of bilayer cuprate superconductors in the SC state based on the kinetic energy driven $\mathrm{SC}$ mechanism 19 . Our results show that the electron spectrum of bilayer cuprate superconductors is split into the bonding and antibonding components by the BS, then the observed PDH structure around the $[\pi, 0]$ point is mainly caused by this BS, with the SC peak being related to the antibonding component, and the hump being formed by the bonding component. The spectral weight increases with increasing the doping concentration. In analogy to the two flat bands appeared in the normal state, the antibonding and bonding quasiparticles around the $[\pi, 0]$ point disperse weakly with momentum, in qualitative agreement with the experimental observation on the bilayer cuprate superconductors $2,7,8,9,10,11$. Our these results also show that the bilayer interaction has significant contributions to the electronic structure of bilayer cuprate superconductors in the SC state.

It has been shown from the ARPES experiments 2,14 that the BS has been detected in both normal and SC states, and then the electron spectral functions display the double-peak structure in the normal state and PDH structure in the SC state. Recently, we ${ }^{22}$ have studied the electron spectrum of bilayer cuprate superconductors in the normal state, and shown that the double-peak structure in the electron spectrum in the normal state is dominated by the BS. On the other hand, although the antibonding and bonding SC peaks have different dispersions, the antibonding and bonding parts have the same d-wave SC gap amplitude as mentioned above. Incorporating our previous discussions for the normal state case $\mathrm{e}^{22}$ and the present studies for the SC state case, we therefore find that the one of the important roles of the interlayer coherent hopping (2) is to split the electron spectrum of the bilayer system into the bonding and antibonding components in both normal and SC states. As a consequence, the well pronounced PDH structure of bilayer cuprate superconductors in the SC state and double-peak structure in the normal state are mainly caused by the BS.

\section{Acknowledgments}

The authors would like to thank Dr. H. Guo and Dr. L. Cheng for the helpful discussions. This work was supported by the National Natural Science Foundation of China under Grant No. 90403005, and the funds from the Ministry of Science and Technology of China under Grant Nos. 2006CB601002 and 2006CB921300. 


\section{APPENDIX A: DRESSED HOLON BCS TYPE NORMAL AND ANOMALOUS GREEN'S FUNCTIONS IN BILAYER CUPRATE SUPERCONDUCTORS}

In the single layer case, it has been shown $\frac{19}{1}$ that the dressed holon-spin interactions from the kinetic energy terms of the $t-t^{\prime}-J$ model are quite strong, and in the case without AFLRO, these interactions can induce the dressed holon pairing state (then the electron Cooper pairing state) by exchanging spin excitations in the higher power of the doping concentration. Following their discussions $\frac{18,19}{19}$, we obtain in terms of Eliashberg's strong coupling theory $\underline{31}$ that the self-consistent equations that satisfied by the full dressed holon normal and anomalous Green's functions in the bilayer system in the $\mathrm{SC}$ state as,

$$
\begin{aligned}
& g(\mathbf{k}, \omega)=g^{(0)}(\mathbf{k}, \omega)+g^{(0)}(\mathbf{k}, \omega)\left[\Sigma_{1}^{(h)}(\mathbf{k}, \omega) g(\mathbf{k}, \omega)\right. \\
&\left.-\Sigma_{2}^{(h)}(-\mathbf{k},-\omega) \Im^{\dagger}(\mathbf{k}, \omega)\right],(\mathrm{A} 1 \mathrm{a}) \\
& \Im^{\dagger}(\mathbf{k}, \omega)=g^{(0)}(-\mathbf{k},-\omega)\left[\Sigma_{1}^{(h)}(-\mathbf{k},-\omega) \Im^{\dagger}(-\mathbf{k},-\omega)\right. \\
&\left.+\Sigma_{2}^{(h)}(-\mathbf{k},-\omega) g(\mathbf{k}, \omega)\right], \quad(\mathrm{A} 1 \mathrm{~b})
\end{aligned}
$$

respectively, where the MF dressed holon normal Green's function ${ }^{22} g^{(0)}(\mathbf{k}, \omega)=g_{L}^{(0)}(\mathbf{k}, \omega)+\sigma_{x} g_{T}^{(0)}(\mathbf{k}, \omega)$, with the longitudinal and transverse parts are evaluated as $g_{L}^{(0)}(\mathbf{k}, \omega)=(1 / 2) \sum_{\nu=1,2}\left(\omega-\xi_{\nu \mathbf{k}}\right)^{-1}$ and $g_{T}^{(0)}(\mathbf{k}, \omega)=$ $(1 / 2) \sum_{\nu=1,2}(-1)^{\nu+1}\left(\omega-\xi_{\nu \mathbf{k}}\right)^{-1}$, respectively, while the dressed holon self-energy functions $\Sigma_{1}^{(h)}(\mathbf{k}, \omega)=$ $\Sigma_{1 L}^{(h)}(\mathbf{k}, \omega)+\sigma_{x} \Sigma_{1 T}^{(h)}(\mathbf{k}, \omega)$ and $\Sigma_{2}^{(h)}(\mathbf{k}, \omega)=\Sigma_{2 L}^{(h)}(\mathbf{k}, \omega)+$ $\sigma_{x} \Sigma_{2 T}^{(h)}(\mathbf{k}, \omega)$, with the corresponding longitudinal and transverse parts have been given in Eq. (6).

In the previous discussions of the electronic struc- ture for the single layer cuprate superconductors in the SC state $\frac{18}{}$, it has been shown the self-energy function $\Sigma_{2}^{(h)}(\mathbf{k}, \omega)$ describes the effective dressed holon pair gap function, while the self-energy function $\Sigma_{1}^{(h)}(\mathbf{k}, \omega)$ describes the quasiparticle coherence. Since $\Sigma_{2}^{(h)}(\mathbf{k}, \omega)$ is an even function of $\omega$, while $\Sigma_{1}^{(h)}(\mathbf{k}, \omega)$ is not, therefore for the convenience, the self-energy function $\Sigma_{1}^{(h)}(\mathbf{k}, \omega)$ can be broken up into its symmetric and antisymmetric parts as, $\Sigma_{1}^{(h)}(\mathbf{k}, \omega)=\Sigma_{1 e}^{(h)}(\mathbf{k}, \omega)+\omega \Sigma_{1 o}^{(h)}(\mathbf{k}, \omega)$, then both $\Sigma_{1 e}^{(h)}(\mathbf{k}, \omega)$ and $\Sigma_{1 o}^{(h)}(\mathbf{k}, \omega)$ are even functions of $\omega$. Now we can define the dressed holon quasiparticle coherent weights in the present bilayer system as $Z_{h F 1}^{-1}(\mathbf{k}, \omega)=$ $1-\Sigma_{1 L}^{(h o)}(\mathbf{k}, \omega)$ and $Z_{h F 2}^{-1}(\mathbf{k}, \omega)=\Sigma_{1 T}^{(h o)}(\mathbf{k}, \omega)$. As in the single layer case $\frac{18}{\underline{18}}$, we only discuss the low-energy behavior of the electronic structure of bilayer cuprate superconductors, which means that the effective dressed holon pair gap functions and quasiparticle coherent weights can be discussed in the static limit, i.e., $\bar{\Delta}_{h}(\mathbf{k})=$ $\left.\Sigma_{2}^{(h)}(\mathbf{k}, \omega)\right|_{\omega=0}=\bar{\Delta}_{h L}(\mathbf{k})+\sigma_{x} \bar{\Delta}_{h T}(\mathbf{k}), Z_{h F 1}^{-1}(\mathbf{k})=1-$ $\left.\Sigma_{1 L}^{(h o)}(\mathbf{k}, \omega)\right|_{\omega=0}$ and $Z_{h F 2}^{-1}(\mathbf{k})=\left.\Sigma_{1 T}^{(h o)}(\mathbf{k}, \omega)\right|_{\omega=0}$. As in the single layer case $\stackrel{18}{ }$, although $Z_{h F 1}(\mathbf{k})$ and $Z_{h F 2}(\mathbf{k})$ still are a function of $\mathbf{k}$, the wave vector dependence may be unimportant. This followed from the ARPES experiments ${ }^{2}$ that in the SC-state of bilayer cuprate superconductors, the lowest energy states are located at the $[\pi, 0]$ point, which indicates that the majority contribution for the electron spectrum comes from the $[\pi, 0]$ point. In this case, the wave vector $\mathbf{k}$ in $Z_{h F 1}(\mathbf{k})$ and $Z_{h F 2}(\mathbf{k})$ can be chosen as $Z_{h F 1}^{-1}=1-\left.\Sigma_{1 L}^{(h o)}(\mathbf{k})\right|_{\mathbf{k}=[\pi, 0]}$ and $Z_{h F 2}^{-1}=\left.\Sigma_{1 T}^{(h o)}(\mathbf{k})\right|_{\mathbf{k}=[\pi, 0]}$. With the help of the above discussions, the corresponding longitudinal and transverse parts of the dressed holon normal and anomalous Green's functions in Eqs. (A1a) and (A1b) now can be obtained explicitly as,

$$
\begin{aligned}
g_{L}(\mathbf{k}, \omega) & =\frac{1}{2} \sum_{\nu=1,2} Z_{h F A}^{(\nu)}\left(\frac{U_{h \nu \mathbf{k}}^{2}}{\omega-E_{h \nu \mathbf{k}}}+\frac{V_{h \nu \mathbf{k}}^{2}}{\omega+E_{h \nu \mathbf{k}}}\right), \\
g_{T}(\mathbf{k}, \omega) & =\frac{1}{2} \sum_{\nu=1,2}(-1)^{\nu+1} Z_{h F A}^{(\nu)}\left(\frac{U_{h \nu \mathbf{k}}^{2}}{\omega-E_{h \nu \mathbf{k}}}+\frac{V_{h \nu \mathbf{k}}^{2}}{\omega+E_{h \nu \mathbf{k}}}\right), \\
\Im_{L}^{\dagger}(\mathbf{k}, \omega) & =-\frac{1}{2} \sum_{\nu=1,2} Z_{h F A}^{(\nu)} \frac{\bar{\Delta}_{h z}^{(\nu)}(\mathbf{k})}{2 E_{h \nu \mathbf{k}}}\left(\frac{1}{\omega-E_{h \nu \mathbf{k}}}-\frac{1}{\omega+E_{h \nu \mathbf{k}}}\right), \\
\Im_{T}^{\dagger}(\mathbf{k}, \omega) & =-\frac{1}{2} \sum_{\nu=1,2}(-1)^{\nu+1} Z_{h F A}^{(\nu)} \frac{\bar{\Delta}_{h z}^{(\nu)}(\mathbf{k})}{2 E_{h \nu \mathbf{k}}}\left(\frac{1}{\omega-E_{h \nu \mathbf{k}}}-\frac{1}{\omega+E_{h \nu \mathbf{k}}}\right),
\end{aligned}
$$

with the dressed holon effective gap parameters and quasiparticle coherent weights satisfy the following four equations, 


$$
\begin{aligned}
& \bar{\Delta}_{h L}=-\frac{4}{32 N^{3}} \sum_{\mathbf{k}, \mathbf{q}, \mathbf{p}} \sum_{\nu, \nu^{\prime}, \nu^{\prime \prime}} \gamma_{\mathbf{k}-\mathbf{p}+\mathbf{q}}^{(d)} C_{\nu \nu^{\prime \prime}}(\mathbf{k}+\mathbf{q}) \frac{Z_{h F A}^{\left(\nu^{\prime \prime}\right)} B_{\nu^{\prime} \mathbf{p}} B_{\nu \mathbf{q}}}{\omega_{\nu^{\prime} \mathbf{p}} \omega_{\nu \mathbf{q}}} \bar{\Delta}_{h z}^{\left(\nu^{\prime \prime}\right)}(\mathbf{k}) \\
& \times\left(\frac{F_{\nu \nu^{\prime} \nu^{\prime \prime}}^{(1)}(\mathbf{q}, \mathbf{p})+F_{\nu \nu^{\prime} \nu^{\prime \prime}}^{(2)}(\mathbf{k}, \mathbf{q}, \mathbf{p})}{\left[\omega_{\nu^{\prime}} \mathbf{p}-\omega_{\nu \mathbf{q}}\right]^{2}-E_{h \nu^{\prime \prime} \mathbf{k}}^{2}}+\frac{F_{\nu \nu^{\prime} \nu^{\prime \prime}}^{(3)}(\mathbf{q}, \mathbf{p})+F_{\nu \nu^{\prime} \nu^{\prime \prime}}^{(4)}(\mathbf{k}, \mathbf{q}, \mathbf{p})}{\left[\omega_{\nu^{\prime} \mathbf{p}}+\omega_{\nu \mathbf{q}}\right]^{2}-E_{h \nu^{\prime \prime} \mathbf{k}}^{2}}\right), \\
& \bar{\Delta}_{h T}=-\frac{1}{32 N^{3}} \sum_{\mathbf{k}, \mathbf{q}, \mathbf{p}} \sum_{\nu, \nu^{\prime}, \nu^{\prime \prime}}(-1)^{\nu+\nu^{\prime}+\nu^{\prime \prime}+1} C_{\nu \nu^{\prime \prime}}(\mathbf{k}+\mathbf{q}) \frac{Z_{h F A}^{\left(\nu^{\prime \prime}\right)} B_{\nu^{\prime} \mathbf{p}} B_{\nu \mathbf{q}}}{\omega_{\nu^{\prime} \mathbf{p}} \omega_{\nu \mathbf{q}}} \bar{\Delta}_{h z}^{\left(\nu^{\prime \prime}\right)}(\mathbf{k}) \\
& \times\left(\frac{F_{\nu \nu^{\prime} \prime^{\prime \prime}}^{(1)}(\mathbf{q}, \mathbf{p})+F_{\nu \nu^{\prime}{ }^{\prime \prime}}^{(2)}(\mathbf{k}, \mathbf{q}, \mathbf{p})}{\left[\omega_{\nu^{\prime} \mathbf{p}}-\omega_{\nu \mathbf{q}}\right]^{2}-E_{h \nu^{\prime \prime} \mathbf{k}}^{2}}+\frac{F_{\nu \nu^{\prime} \nu^{\prime \prime}}^{(3)}(\mathbf{q}, \mathbf{p})+F_{\nu \nu^{\prime} \nu^{\prime \prime}}^{(4)}(\mathbf{k}, \mathbf{q}, \mathbf{p})}{\left[\omega_{\nu^{\prime} \mathbf{p}}+\omega_{\nu \mathbf{q}}\right]^{2}-E_{h \nu^{\prime \prime} \mathbf{k}}^{2}}\right), \\
& \frac{1}{Z_{h F A}^{(1)}}=1+\frac{1}{32 N^{2}} \sum_{\mathbf{q}, \mathbf{p}} \sum_{\nu, \nu^{\prime}, \nu^{\prime \prime}}\left[1+(-1)^{\nu+\nu^{\prime}+\nu^{\prime \prime}+1}\right] C_{\nu \nu^{\prime \prime}}\left(\mathbf{p}+\mathbf{k}_{\mathbf{0}}\right) \frac{Z_{h F A}^{\left(\nu^{\prime \prime}\right)} B_{\nu^{\prime} \mathbf{p}} B_{\nu \mathbf{q}}}{\omega_{\nu^{\prime} \mathbf{p}} \omega_{\nu \mathbf{q}}} \\
& \times\left(\frac{H_{\nu \nu^{\prime} \nu^{\prime \prime}}^{(1)}(\mathbf{q}, \mathbf{p})}{\left[\omega_{\nu^{\prime} \mathbf{p}}-\omega_{\nu \mathbf{q}}+E_{\left.h \nu^{\prime \prime} \mathbf{p}-\mathbf{q}+\mathbf{k}_{\mathbf{0}}\right]^{2}}\right.}+\frac{H_{\nu \nu^{\prime} \nu^{\prime \prime}}^{(2)}(\mathbf{q}, \mathbf{p})}{\left[\omega_{\nu^{\prime} \mathbf{p}}-\omega_{\nu \mathbf{q}}-E_{h \nu^{\prime \prime} \mathbf{p}-\mathbf{q}+\mathbf{k}_{0}}\right]^{2}}\right.
\end{aligned}
$$

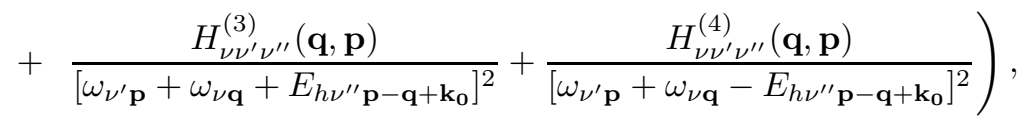

$$
\begin{aligned}
& \frac{1}{Z_{h F A}^{(2)}}=1+\frac{1}{32 N^{2}} \sum_{\mathbf{q}, \mathbf{p}} \sum_{\nu, \nu^{\prime}, \nu^{\prime \prime}}\left[1-(-1)^{\nu+\nu^{\prime}+\nu^{\prime \prime}+1}\right] C_{\nu \nu^{\prime \prime}}\left(\mathbf{p}+\mathbf{k}_{\mathbf{0}}\right) \frac{Z_{h F A}^{\left(\nu^{\prime \prime}\right)} B_{\nu^{\prime} \mathbf{p}} B_{\nu \mathbf{q}}}{\omega_{\nu^{\prime} \mathbf{p}} \omega_{\nu \mathbf{q}}} \\
& \times\left(\frac{H_{\nu \nu^{\prime} \nu^{\prime \prime}}^{(1)}(\mathbf{q}, \mathbf{p})}{\left[\omega_{\nu^{\prime} \mathbf{p}}-\omega_{\nu \mathbf{q}}+E_{\left.h \nu^{\prime \prime} \mathbf{p}-\mathbf{q}+\mathbf{k}_{\mathbf{0}}\right]^{2}}\right.}+\frac{H_{\nu \nu^{\prime} \nu^{\prime \prime}}^{(2)}(\mathbf{q}, \mathbf{p})}{\left[\omega_{\nu^{\prime} \mathbf{p}}-\omega_{\nu \mathbf{q}}-E_{\left.h \nu^{\prime \prime} \mathbf{p}-\mathbf{q}+\mathbf{k}_{\mathbf{0}}\right]^{2}}\right.}\right. \\
& +\frac{H_{\nu \nu^{\prime} \nu^{\prime \prime}}^{(3)}(\mathbf{q}, \mathbf{p})}{\left[\omega_{\nu^{\prime} \mathbf{p}}+\omega_{\nu \mathbf{q}}+E_{\left.h \nu^{\prime \prime} \mathbf{p}-\mathbf{q}+\mathbf{k}_{\mathbf{o}}\right]^{2}}\right.}+\frac{H_{\nu \nu^{\prime} \nu^{\prime \prime}}^{(4)}(\mathbf{q}, \mathbf{p})}{\left[\omega_{\nu^{\prime} \mathbf{p}}+\omega_{\nu \mathbf{q}}-E_{\left.h \nu^{\prime \prime} \mathbf{p}-\mathbf{q}+\mathbf{k}_{\mathbf{0}}\right]^{2}}\right)},
\end{aligned}
$$

where $C_{\nu \nu^{\prime \prime}}(\mathbf{k})=\left[Z\left(t \gamma_{\mathbf{k}}-t^{\prime} \gamma_{\mathbf{k}}^{\prime}\right)+(-1)^{\nu+\nu^{\prime \prime}} t_{\perp}(\mathbf{k})\right]^{2}$, $F_{\nu \nu^{\prime} \nu^{\prime \prime}}^{(1)}(\mathbf{q}, \mathbf{p})=n_{B}\left(\omega_{\nu \mathbf{q}}\right)+n_{B}\left(\omega_{\nu^{\prime}} \mathbf{p}\right)+2 n_{B}\left(\omega_{\nu \mathbf{q}}\right) n_{B}\left(\omega_{\nu^{\prime} \mathbf{p}}\right)$, $F_{\nu \nu^{\prime} \nu^{\prime \prime}}^{(2)}(\mathbf{k}, \mathbf{q}, \mathbf{p})=\left[2 n_{F}\left(E_{h \nu^{\prime \prime} \mathbf{k}}\right)-1\right]\left[\omega_{\nu^{\prime} \mathbf{p}}-\right.$ $\left.\omega_{\nu \mathbf{q}}\right]\left[n_{B}\left(\omega_{\nu \mathbf{q}}\right)-n_{B}\left(\omega_{\nu^{\prime} \mathbf{p}}\right)\right] / E_{h \nu^{\prime \prime} \mathbf{k}}, \quad F_{\nu \nu^{\prime} \nu^{\prime \prime}}^{(3)}(\mathbf{q}, \mathbf{p})=$ $1+n_{B}\left(\omega_{\nu \mathbf{q}}\right)+n_{B}\left(\omega_{\nu^{\prime} \mathbf{p}}\right)+2 n_{B}\left(\omega_{\nu \mathbf{q}}\right) n_{B}\left(\omega_{\nu^{\prime} \mathbf{p}}\right)$, $F_{\nu \nu^{\prime} \nu^{\prime \prime}}^{(4)}(\mathbf{k}, \mathbf{q}, \mathbf{p})=\left[2 n_{F}\left(E_{h \nu^{\prime \prime} \mathbf{k}}\right)-1\right]\left[\omega_{\nu^{\prime} \mathbf{p}}+\omega_{\nu \mathbf{q}}\right][1+$ $\left.n_{B}\left(\omega_{\nu \mathbf{q}}\right)+n_{B}\left(\omega_{\nu^{\prime} \mathbf{p}}\right)\right] / E_{h \nu^{\prime \prime} \mathbf{k}}, \quad H_{\nu \nu^{\prime} \nu^{\prime \prime}}^{(1)}(\mathbf{q}, \mathbf{p})=$

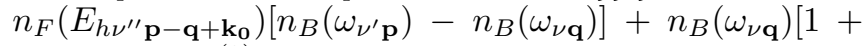

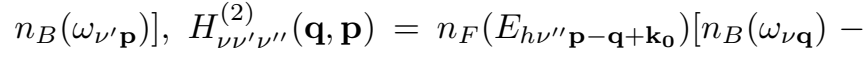

$\left.n_{B}\left(\omega_{\nu^{\prime}} \mathbf{p}\right)\right]+n_{B}\left(\omega_{\nu^{\prime} \mathbf{p}}\right)\left[1+n_{B}\left(\omega_{\nu \mathbf{q}}\right)\right], \quad H_{\nu \nu^{\prime} \nu^{\prime \prime}}^{(3)}(\mathbf{q}, \mathbf{p})=$

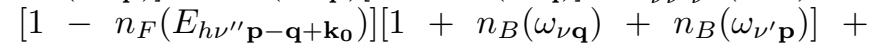
$n_{B}\left(\omega_{\nu \mathbf{q}}\right) n_{B}\left(\omega_{\nu^{\prime}} \mathbf{p}\right), H_{\nu \nu^{\prime} \nu^{\prime \prime}}^{(4)}(\mathbf{q}, \mathbf{p})=n_{F}\left(E_{h \nu^{\prime \prime} \mathbf{p}-\mathbf{q}+\mathbf{k}_{\mathbf{o}}}\right)[1+$ $\left.n_{B}\left(\omega_{\nu \mathbf{q}}\right)+n_{B}\left(\omega_{\nu^{\prime} \mathbf{p}}\right)\right]+n_{B}\left(\omega_{\nu \mathbf{q}}\right) n_{B}\left(\omega_{\nu^{\prime} \mathbf{p}}\right)$, and $\mathbf{k}_{0}=[\pi, 0]$. These four equations must be solved self-consistently in combination with other equations as in the single layer $\operatorname{case}^{\frac{18.19}{2}}$, then all order parameters, decoupling parameter $\alpha$, and chemical potential $\mu$ are determined by the self-consistent calculation.
1 See, e.g., M.A.Kastner, R.J. Birgeneau, G. Shirane, and Y. Endoh, Rev. Mod. Phys. 70, 897 (1998), and references therein.

2 See, e.g., A. Damascelli, Z. Hussain, and Z.-X. Shen, Rev. Mod. Phys. 75, 473 (2003), and references therein.

3 See, e.g., J. Campuzano, M. Norman, and M. Randeira, in Physics of Superconductors, vol. II, edited by K. Bennemann and J. Ketterson (Springer, Berlin Heidelberg New York, 2004), p. 167, and references therein.

4 J. Campuzano, H. Ding, M. R. Norman, M. Randeira, A. F. Bellman, T. Yokoya, T. Takahashi, H. KatayamaYoshida, T. Mochiku, and K. Kadowaki, Phys. Rev. B 53, R14737 (2003).

${ }^{5}$ H. Matsui, T. Sato, T. Takahashi, S.-C. Wang, H.-B. Yang,
H. Ding, T. Fujii, T. Watanabe, and A. Matsuda, Phys. Rev. Lett. 90, 217002 (2003).

6 J.R. Schrieffer, Theory of Superconductivity, Benjamin, New York, 1964.

7 D.S. Dessau, B.O. Wells, Z.-X. Shen, W.E. Spicer, A.J. Arko, R.S. List, D.B. Mitzi, and A. Kapitulnik, Phys. Rev. Lett. 66, 2160 (1991); Y. Hwu, L. Lozzi, M. Marsi, S. La Rosa, M. Winokur, P. Davis, M. Onellion, H. Berger, F. Gozzo, F. Lévy, and G. Margaritondo, Phys. Rev. Lett. 67, 2573 (1991).

8 Mohit Randeria, Hong Ding, J-C. Campuzano, A. Bellman, G. Jennings, T. Yokoya, T. Takahashi, H. KatayamaYoshida, T. Mochiku, and K. Kadowaki, Phys. Rev. Lett. 74, 4951 (1995); H. Ding, T. Yokoya, J-C. Campuzano, T. 
Takahashi, M. Randeria, M. R. Norman, T. Mochiku, K. Kadowaki, and J. Giapintzakis, Nature 382, 51 (1996).

9 A.V. Fedorov, T. Valla, P.D. Johnson, Q. Li, G.D. Gu, and N. Koshizuka, Phys. Rev. Lett. 82, 2179 (1999).

10 D.H. Lu, D.L. Feng, N.P. Armitage, K.M. Shen, A. Damascelli, C. Kim, F. Ronning, Z.-X. Shen, D.A. Bonn, R. Liang, W.N. Hardy, A.I. Rykov, and S. Tajima, Phys. Rev. Lett. 86, 4370 (2001).

11 T. Sato, H. Matsui, S. Nishina, T. Takahashi, T. Fujii, T. Watanabe, and A. Matsuda, Phys. Rev. Lett. 89, 67005 (2002); D.L. Feng, A. Damascelli, K.M. Shen, N. Motoyama, D.H. Lu, H. Eisaki, K. Shimizu, J.-i. Shimoyama, K. Kishio, N. Kaneko, M. Greven, G.D. Gu, X.J. Zhou, C. Kim, F. Ronning, N.P. Armitage, and Z.-X Shen, Phys. Rev. Lett. 88, 107001 (2002).

12 J.C. Campuzano, H. Ding, M.R. Norman, H.M. Fretwell, M. Randeria, A. Kaminski, J. Mesot, T. Takeuchi, T. Sato, T. Yokoya, T. Takahashi, T. Mochiku, K. Kadowaki, P. Guptasarma, D.G. Hinks, Z. Konstantinovic, Z.Z. Li, and H. Raffy, Phys. Rev. Lett. 83, 3709 (1999); M.R. Norman, H. Ding, J.C. Campuzano, T. Takeuchi, M. Randeria, T. Yokoya, T. Takahashi, T. Mochiku, and K. Kadowaki, Phys. Rev. Lett. 79, 3506 (1997).

13 A.A. Kordyuk, S.V. Borisenko, T.K. Kim, K.A. Nenkov, M. Knupfer, J. Fink, M.S. Golden, H. Berger, and R. Follath, Phys. Rev. Lett. 89, 077003 (2002); A.D. Gromko, Y.-D. Chuang, A.V. Fedorov, Y. Aiura, Y. Yamaguchi, K. Oka, Yoichi Ando, D.S. Dessau, cond-mat/0205385

14 D.L. Feng, N.P. Armitage, D.H. Lu, A. Damascelli, J.P. $\mathrm{Hu}$, P. Bogdanov, A. Lanzara, F. Ronning, K.M. Shen, H. Eisaki, C. Kim, Z.-X. Shen, J.-i. Shimoyama, and K. Kishio, Phys. Rev. Lett. 86, 5550 (2001).

15 Y.-D. Chuang, A.D. Gromko, A. Fedorov, Y. Aiura, K. Oka, Yoichi Ando, H. Eisaki, S.I. Uchida, and D.S. Dessau, Phys. Rev. Lett. 87, 117002 (2001); P.V. Bogdanov, A. Lanzara, X.J. Zhou, S.A. Kellar, D.L. Feng, E.D. Lu, H. Eisaki, J.-I. Shimoyama, K. Kishio, Z. Hussain, and Z.X. Shen, Phys. Rev. B 64, 180505(R) (2001).

16 S.V. Borisenko, A.A. Kordyuk, T.K. Kim, S. Legner, K.A. Nenkov, M. Knupfer, M.S. Golden, J. Fink, H. Berger, and R. Follath, Phys. Rev. B 66, 140509(R) (2002).

17 D.L. Feng, C. Kim, H. Eisaki, D.H. Lu, A. Damascelli, K.M. Shen, F. Ronning, N.P. Armitage, N. Kaneko1, M. Greven, J.-i. Shimoyama, K. Kishio, R. Yoshizaki, G.D. Gu, and Z.-X. Shen, Phys. Rev. B 65, 220501(R) (2002); A.A. Kordyuk, S.V. Borisenko, M.S. Golden, S. Legner, K.A. Nenkov, M. Knupfer, J. Fink, H. Berger, L. Forró, and R. Follath, Phys. Rev. B 66, 014502 (2002); Y.-D. Chuang, A.D. Gromko, A.V. Fedorov, Y. Aiura, K. Oka, Yoichi Ando, D.S. Dessau, cond-mat/0107002.
18 Huaiming Guo and Shiping Feng, Phys. Lett. A 361, 382 (2007); Shiping Feng and Tianxing Ma, Phys. Lett. A 350, 138 (2006).

19 Shiping Feng, Phys. Rev. B68, 184501 (2003); Shiping Feng, Tianxing Ma, and Huaiming Guo, Physica C 436, 14 (2006).

20 A.A. Kordyuk, S.V. Borisenko, M. Knupfer, and J. Fink, Phys. Rev. B 67, 064504 (2003); A.A. Kordyuk and S.V. Borisenko, Low Temp. Phys. 32, 298 (2006).

21 M. Mori, T. Tohyama, and S. Maekawa, Phys. Rev. B 66, 064502 (2002).

${ }^{22} \mathrm{Yu}$ Lan, Jihong Qin, and Shiping Feng, Phys. Rev. B 75, 134513 (2007).

${ }^{23}$ C. Kim, P.J. White, Z.-X. Shen, T. Tohyama, Y. Shibata, S. Maekawa, B.O. Wells, Y.J. Kim, R.J. Birgeneau, and M.A. Kastner, Phys. Rev. Lett. 80, 4245 (1998).

24 O.K. Anderson, A.I. Liechtenstein, O. Jepsen, and F. Paulsen, J. Phys. Chem. Solids 56, 1573 (1995); A.I. Liechtenstein, O. Gunnarsson, O.K. Anderson, and R.M. Martin, Phys. Rev. B 54, 12505 (1996); S. Chakarvarty, A. Sudbo, P.W. Anderson, and S. Strong, Science 261, 337 (1993).

25 Shiping Feng, Jihong Qin, and Tianxing Ma, J. Phys. Condens. Matter 16, 343 (2004); Shiping Feng, Tianxing Ma, and Jihong Qin, Mod. Phys. Lett. B 17, 361 (2003).

26 S. Sorella, G.B. Martins, F. Becca, C. Gazza, L. Capriotti, A. Parola, and E. Dagotto, Phys. Rev. Lett. 88, 117002 (2002).

27 P.W. Anderson, Phys. Rev. Lett. 67, 2092 (1991); Science 288, 480 (2000).

28 P. Dai, H.A. Mook, R.D. Hunt, and F. Dog̃an, Phys. Rev. B 63, 54525 (2001); Ph. Bourges, B. Keimer, S. Pailhés, L.P. Regnault, Y. Sidis, and C. Ulrich, Physica C 424, 45 (2005).

29 M. Arai, T. Nishijima, Y. Endoh, T. Egami, S. Tajima, K. Tomimoto, Y. Shiohara, M. Takahashi, A. Garret, and S.M. Bennington, Phys. Rev. Lett. 83, 608 (1999); S.M. Hayden, H.A. Mook, P. Dai, T.G. Perring, and F. Dog̃an, Nature 429, 531 (2004); C. Stock, W.J. Buyers, R.A. Cowley, P.S. Clegg, R. Coldea, C.D. Frost, R. Liang, D. Peets, D. Bonn, W.N. Hardy, and R.J. Birgeneau, Phys. Rev. B 71, 24522 (2005).

${ }^{30}$ K. Terashima, H. Matsui, D. Hashimoto, T. Sato, T. Takahashi, H. Ding, T. Yamamoto, and K. Kadowaki, Nature Phys. 2, 27 (2006).

31 G.M. Eliashberg, Sov. Phys. JETP 11, 696 (1960); D.J. Scalapino, J.R. Schrieffer, and J.W. Wilkins, Phys. Rev. 148, 263 (1966). 